\title{
Reduction of antibiotic prescriptions for acute respiratory tract infections in primary care: a systematic review
}

\author{
Anna Köchling ${ }^{1,6^{*}}$, Christin Löffler ${ }^{2}$, Stefan Reinsch ${ }^{3}$, Anne Hornung ${ }^{4}$, Femke Böhmer ${ }^{2}$, Attila Altiner ${ }^{2}$
} and Jean-François Chenot ${ }^{5}$

\begin{abstract}
Background: Although most respiratory tract infections (RTIs) are due to viral infections, they cause the majority of antibiotic (Abx) prescriptions in primary care. This systematic review summarises the evidence on the effectiveness of interventions in primary care aiming to reduce Abx prescriptions in patients $\geq 13$ years for acute RTI.

Methods: We searched the databases "MEDLINE/PubMed" and "Cochrane Library" for the period from January 1, 2005, to August 31, 2016, for randomised controlled trials (RCTs) in primary care aiming at the reduction of Abx prescriptions for patients suffering from RTI. Out of 690 search results, 67 publications were retrieved and 17 RCTs were included. We assumed an absolute change of $10 \%$ as minimal important change.

Results: Twelve out of 17 included RCTs showed statistically significant lower Abx prescription rates in the intervention groups, but only six of them reported a clinically relevant reduction according to our definition. Communication skills training (CST) and point-of-care testing (POCT) were the most effective interventions. Pre-intervention Abx prescription rates varied between $13.5 \%$ and $80 \%$ and observed reductions ranged from 1.5 to $23.3 \%$. Studies with post-intervention rates lower than $20 \%$ had no significant effects. Post-intervention observation periods ranged from 2 weeks up to 3. 5 years. The design of the trials was heterogeneous precluding calculation of pooled effect size. The reporting of many RCTs was poor.

Conclusions: CST and POCT alone or as adjunct can reduce antibiotic prescriptions for RTI. Eleven out of 17 trials were not successfully reducing Abx prescription rates according to our definition of minimal important change. However, five of them reported a statistically significant reduction. Trials with initially lower prescription rates were less likely to be successful. Future trials should investigate sustainability of intervention effects for a longer time period. The generalisability of findings was limited due to heterogeneous designs and outcome measures. Therefore, a consensus of designing and reporting of studies aiming at reducing antibiotic prescriptions is urgently needed to generate meaningful evidence.
\end{abstract}

Keywords: Acute respiratory tract infections, Antibiotics, Primary care

\footnotetext{
* Correspondence: anna.koechling@med.uni-rostock.de

${ }^{1}$ Clinic for Psychosomatic Medicine and Psychotherapy, University Medical

Center, Rostock, Germany

${ }^{6}$ Institute of General Practice, University Medical Center, Rostock, Germany

Full list of author information is available at the end of the article
} 


\section{Introduction}

Although most respiratory tract infections (RTIs) are due to viral infections, they cause the majority of antibiotic prescriptions in primary care [1]. Most patients suffering from RTI do not benefit from an antibiotic treatment since severity and duration of the disease are not relevantly altered. On the contrary, many patients experience side effects such as diarrhoea and rash [2]. Additionally, unnecessary antibiotic prescriptions contribute to increasing bacterial resistance to standard antibiotics [3].

Various interventions have been evaluated to reduce antibiotic prescribing for RTI, e.g. public campaigns, distribution of printed educational material or group education meetings [4-6]. A recent systematic review found moderate short-term effects on antibiotic prescribing of interventions facilitating shared decisionmaking [7]. Another global review summarised the effects of antimicrobial stewardship programs in ambulatory care including interventions for all infectious conditions and children. This review found low-strength evidence for interventions including provider and/or patient education, guidelines, delayed prescribing (DP) and computerised clinical decision support systems (CDSS) [8]. It is expected that within the next years, antibiotic stewardship programs will need to be established worldwide [9]. So far, there is still a discussion and uncertainty about which specific elements of interventions lead to high effectiveness and sustainability [10]. Therefore, this review aims to update and summarise current evidence of various interventions in primary care on reducing antibiotic prescription rates (Abx prescription rates) due to acute RTIs in patients $\geq 13$ years.

\section{Methods}

This is a systematic review reported according to the PRISMA Statement [11]. This systematic review was not registered.

\section{Search methods for identification of trials}

The systematic literature search was carried out in MEDLINE/PubMed and the Cochrane Library using the following search terms:

((antibiotic") AND ("respiratory tract infection" OR "respiratory tract infections" OR "res-piratory infection") AND (communication OR training OR "point of care test" OR "rapid strep test" OR "delayed prescribing" OR intervention* OR "electronic decision support" OR "clinical decision support system" OR "clinical decision support systems" OR "shared decision making") AND ("primary care" OR "primary health care" OR "medical care")) OR ("“Anti-Bacterial Agents"[Mesh]) AND ("Respiratory Tract Infections"[Mesh]) AND ("Primary Health Care"[Mesh] OR "Physicians, Primary Care”[Mesh]))
Additionally, the bibliographies of the included trials were screened for relevant intervention trials. We could not search further databases like EMBASE due to limited access and lack of funding.

\section{Inclusion and exclusion criteria}

The literature search included (cluster-) randomised controlled trials (RCTs) investigating the effect of interventions aiming to reduce antibiotic prescriptions for RTIs for patients $\geq 13$ years in primary care settings. We excluded pilot trials and non-randomised trials. Eligible interventions were educational seminars, feedback on prescribing behaviour, patient education, communication skills training (CST) for physicians and diagnostic tools such as point-of-care tests (POCT) or (electronic) CDSS. We did not take public campaigns into account. These address a broad audience and use mass media to raise awareness for the problem of inadequate antibiotic prescribing and its influence on bacterial resistance. As our systematic review focuses on primary care, this type of intervention is not considered.

We investigated the primary outcome of Abx prescription rate as well as the number of antibiotic prescriptions for acute upper/lower RTIs (cough and sore throat). Any reported secondary outcome such as patient's reconsultation rate, days to recovery from RTI, prescribed class of antibiotic, rate of inappropriate antibiotic prescriptions (prescriptions not according to guidelines), Abx prescription rate for specific RTIs or usage of diagnostic devices was of interest to this systematic review.

We only included patients $\geq 13$ years as we know that clinical decision-making in paediatric medicine differs from adults. Further, the communication in dyadic consultations between adults differs from "doctor-parent-child triads" and requests other communication styles [12]. In consequence, we excluded children under 13 years. We included primary care physicians working in ambulatory care. Due to different health systems, we included physicians working in practices or primary care clinics.

We only considered publications written in English, German or French dated January 2005 till July 2016.

\section{Trial selection}

Two reviewers (AK, SR or JFC) independently reviewed the titles, index terms and abstracts of the identified references and rated each paper as potentially relevant or not. Discrepancies were resolved by consensus.

\section{Data extraction}

Relevant information for each trial included into the review was extracted by one reviewer and controlled independently by another one (AK, SR). Discrepancies were resolved by consensus. Three authors had to be 
contacted due to missing or unclear data about the age of included patients [13-15]. Gjelstad et al. offered odds ratios (OR) for antibiotic prescriptions for the age group $\geq 13$ years, but no Abx prescription rate. It was possible to get unpublished $\mathrm{Abx}$ prescription rates for the subgroup of patients $\geq 13$ years [14]. Missing data-like $p$ values or absolute numbers of antibiotic prescriptions-was indicated as "not specified" in our tabular summaries of included RCTs (see Tables 1 and 2).

\section{Summary of Abx prescription rates for acute upper/lower RTI}

If available, antibiotic use was presented as the absolute number of prescribed antibiotics. In addition, relative Abx prescription rates expressed as a percentage-with the corresponding 95\% confidence intervals (=95\% CI) and $p$ values-were indicated as well as the difference of Abx prescription rates between intervention group (IG) and control group (CG) (in percent, OR, relative risks (RR) (Tables 1 and 2). The Abx prescription rates before (T0) and after the intervention (T1 or T2) were shown for trials providing pre-post comparison. As RCTs were heterogeneous in study design and therefore in time points for $\mathrm{T} 1$ or $\mathrm{T} 2$, we decided to not exclude RCTs by defining a binding point of time for T1 or T2. Details of included RCTs can be seen in a tabular summary of study characteristics.

\section{Assessment of risk of bias}

Two researchers (AK with SR, FB or JFC) independently assessed the risk of bias using the Cochrane Collaboration's tool for assessing risk of bias in randomised trials (Fig. 1) [16]. We discussed and resolved discrepancies by consensus.

\section{Evaluation of intervention effect}

Due to the heterogeneity of study designs, outcome measures and Abx prescription rates, we considered a pooled estimate of the effect size with Cohen's $d$ to be inappropriate [17].

There is no consensus on which change in Abx prescribing rates marks a meaningful intervention effect. The difference between current Abx prescription rate and optimal prescription rate reflects the range for reducing antibiotic prescriptions to a meaningful extent. But the generalisability for determining an optimal rate is limited by patient age and condition with different likelihoods for bacterial genesis (pneumonia vs. common cold) [18]. One report from the year 2016 states that $44 \%$ of all ambulatory antibiotic prescriptions in the USA are due to RTIs. It is said that $50 \%$ of these prescriptions are inappropriate leading to an optimal prescribing rate of about $20 \%$ for all RTIs [18]. Another publication suggests a prescribing rate of $10-15 \%$ for acute cough [19].

As a compromise in this complex field and to simplify the overview of clinically relevant reductions in Abx prescriptions, we will consider an absolute difference of $10 \%$ between IG and CG for studies with postintervention as minimal important change. For studies with baseline and follow-up, we regard a difference in differences of $10 \%$ as minimal important change. We acknowledge that this threshold is arbitrary, but a different threshold would not have changed our findings fundamentally.

\section{Results}

Study selection

We searched databases on the 31st of July in 2016 and identified 690 publications. Reviewers independently screened for potentially relevant publications and categorised 215 publications as potentially relevant. After removal of duplicates $(n=84)$, the remaining 151 titles and abstracts were screened for eligibility and discussed by the reviewers. Disagreements between reviewers were resolved by consensus. Major reasons for exclusion were different trial populations, e.g. including children, trials carried out in non-primary care settings and nonrandomised study designs. We excluded 84 out of 151 publications. A total of 67 potentially relevant articles were fully screened for inclusion and exclusion criteria (Fig. 2).

Seventeen trials were finally included in this analysis.

\section{Description of included trials}

Seventeen RCTs met the inclusion criteria and were included in this review (Tables 3 and 4). Thirteen trials were cluster RCTs based on physician-, practice- or educational group levels as clusters [13-15, 20-29]. Four RCTs were randomised at patient level [30-33]. The majority of trials used a two-arm study design [13-15, 20, $24,25,27,28,30-33]$. The remaining trials employed a three-arm [21, 23] or factorial study design [22, 26, 29].

All cluster-randomised trials performed clusteradjusted data analyses. The number of participating physicians ranged from 6 to 573 . Most trials were conducted in Europe [13, 14, 20-22, 27, 28, 31], six trials in North America [15, 23-25, 30, 32] and one in Asia [33]. One trial was a multinational project carried out in six European countries (Belgium, Spain, Wales, England, Poland and the Netherlands) [26]. Published baseline data was available for seven trials [14, 20, 23, 26-29]. Eleven trials assessed the Abx prescription rate right after patients' initial consultation [13, 20, 22, 23, 26-31, 33].

\section{Primary endpoints}

Data on Abx prescription rates was collected directly by physicians $[20,21,26,27,31]$, by pharmacists using 


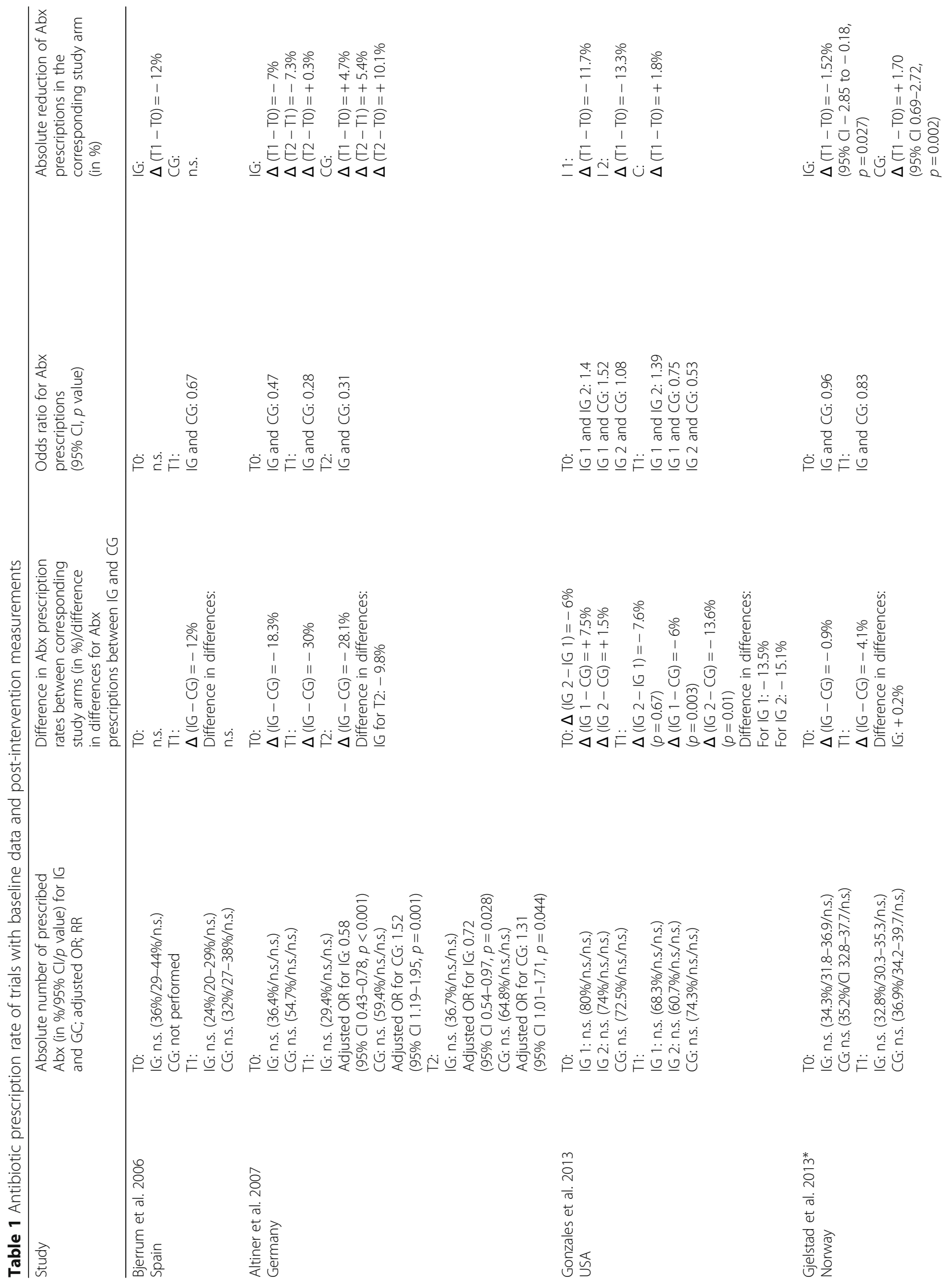




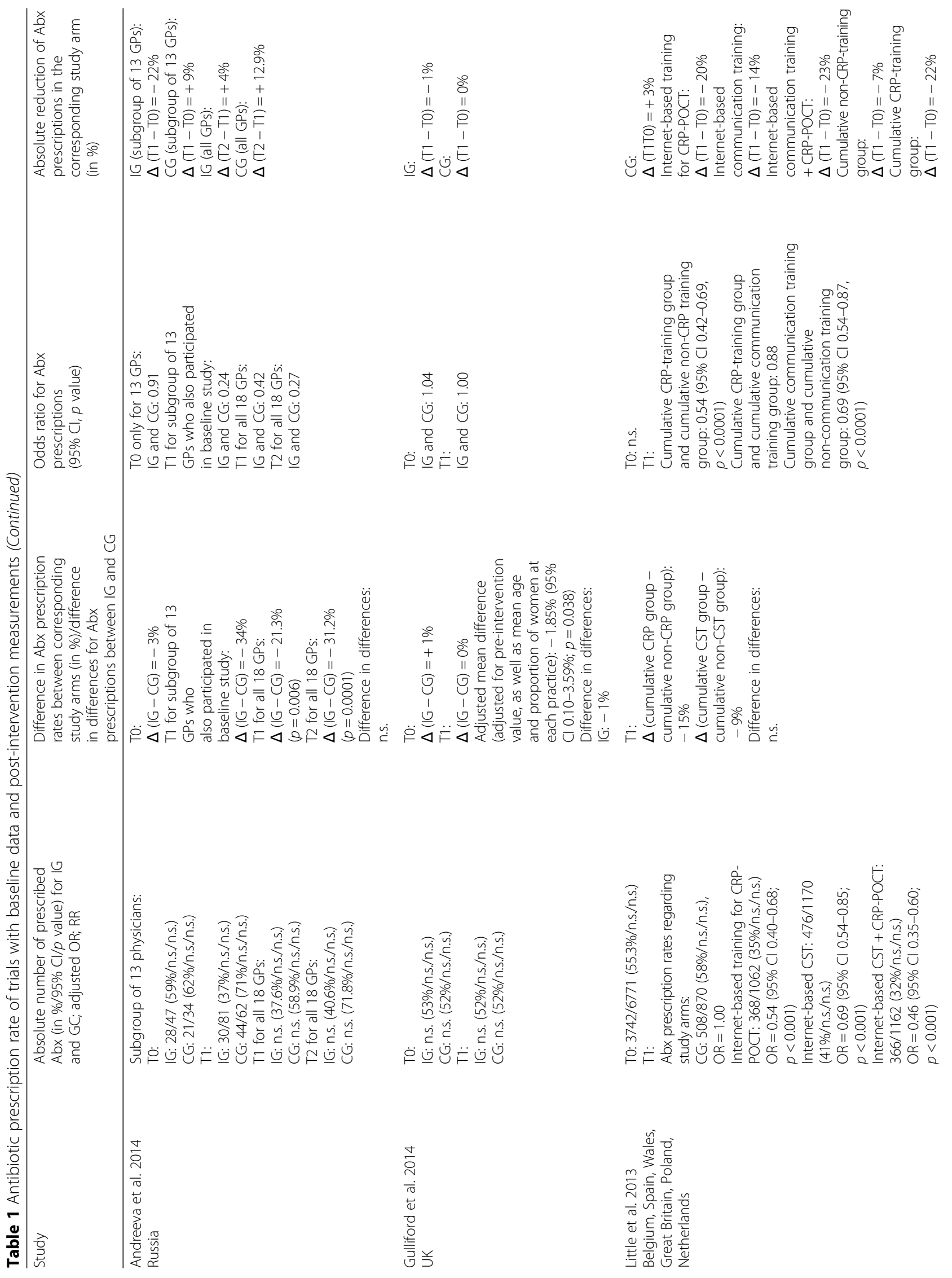




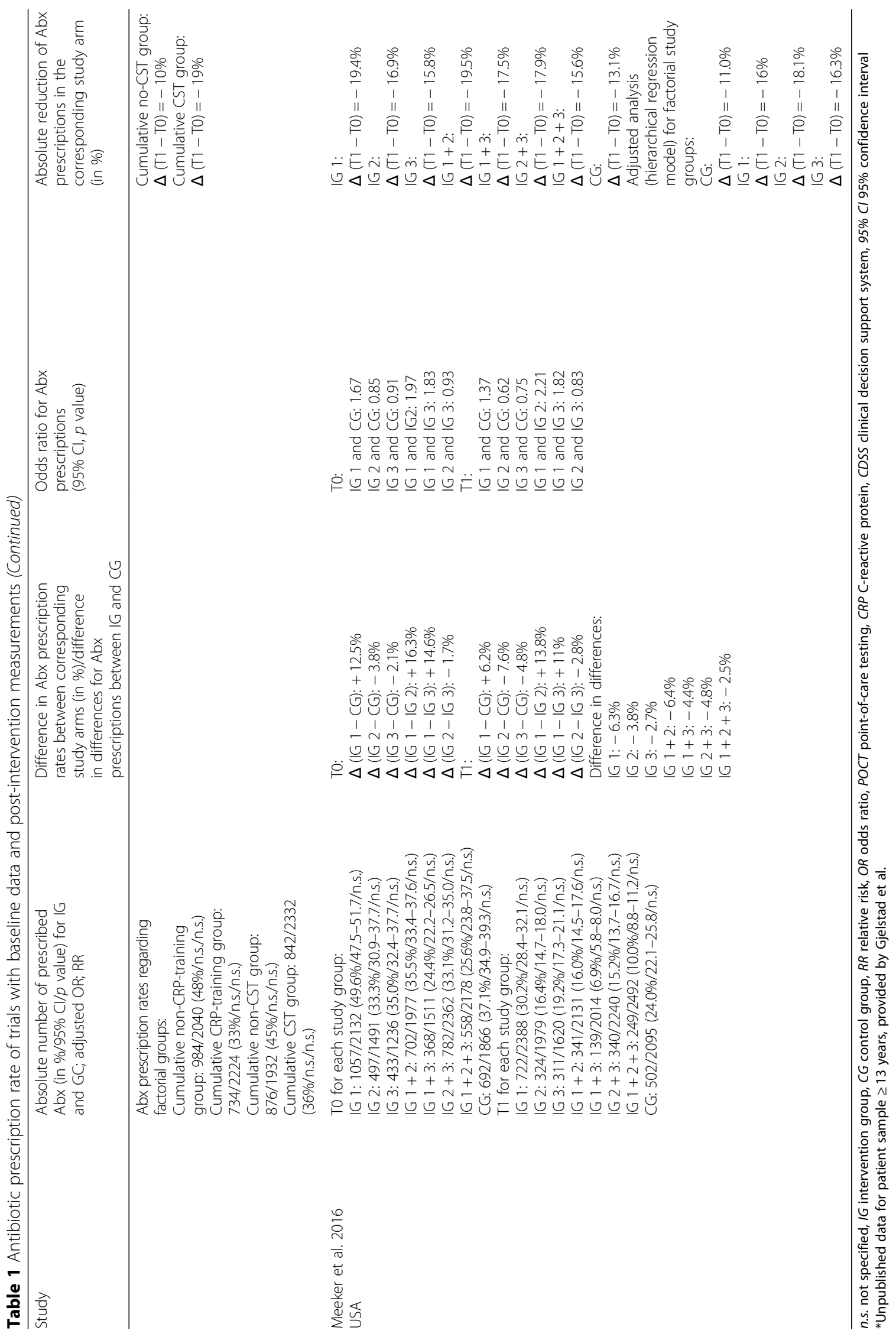




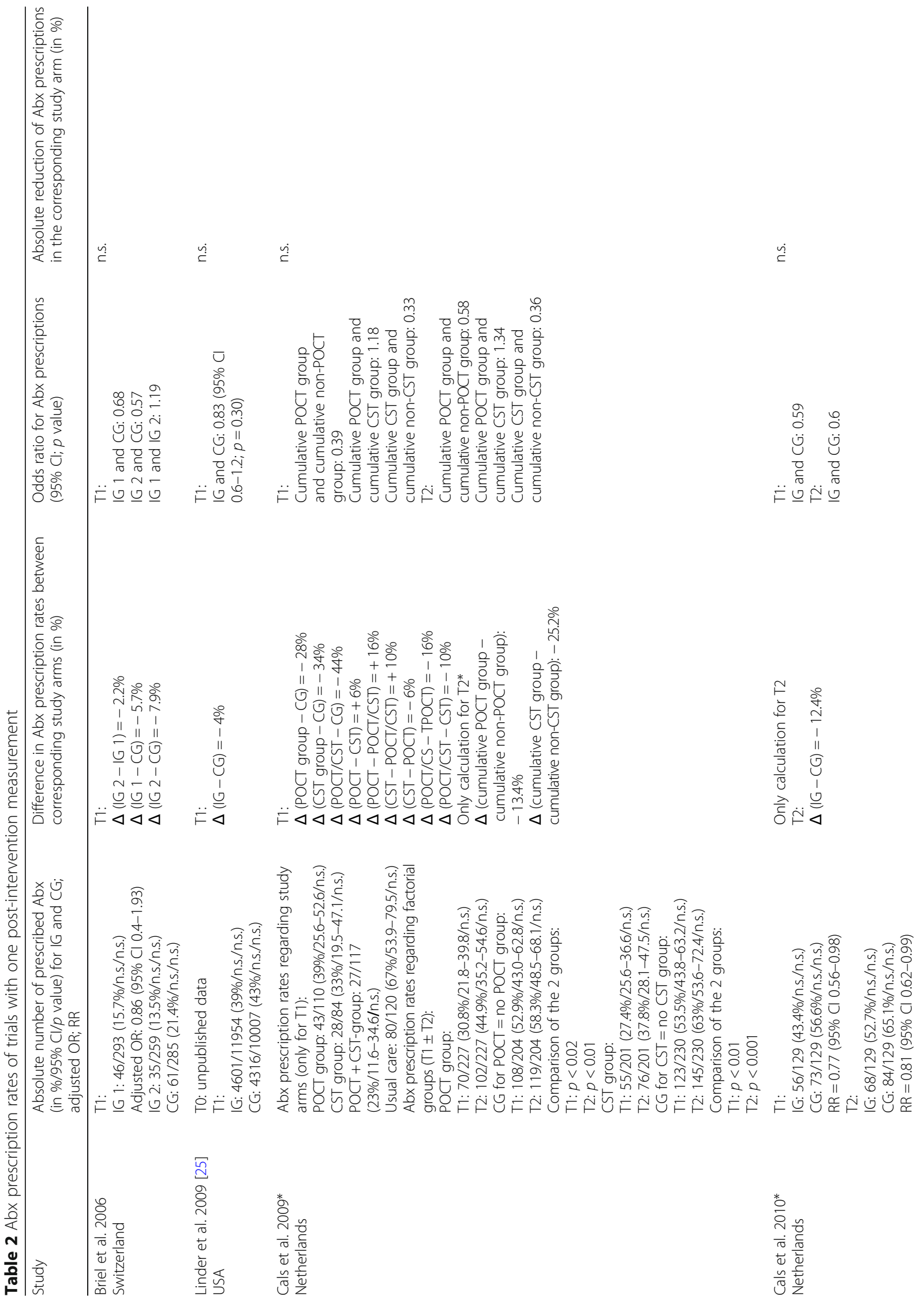




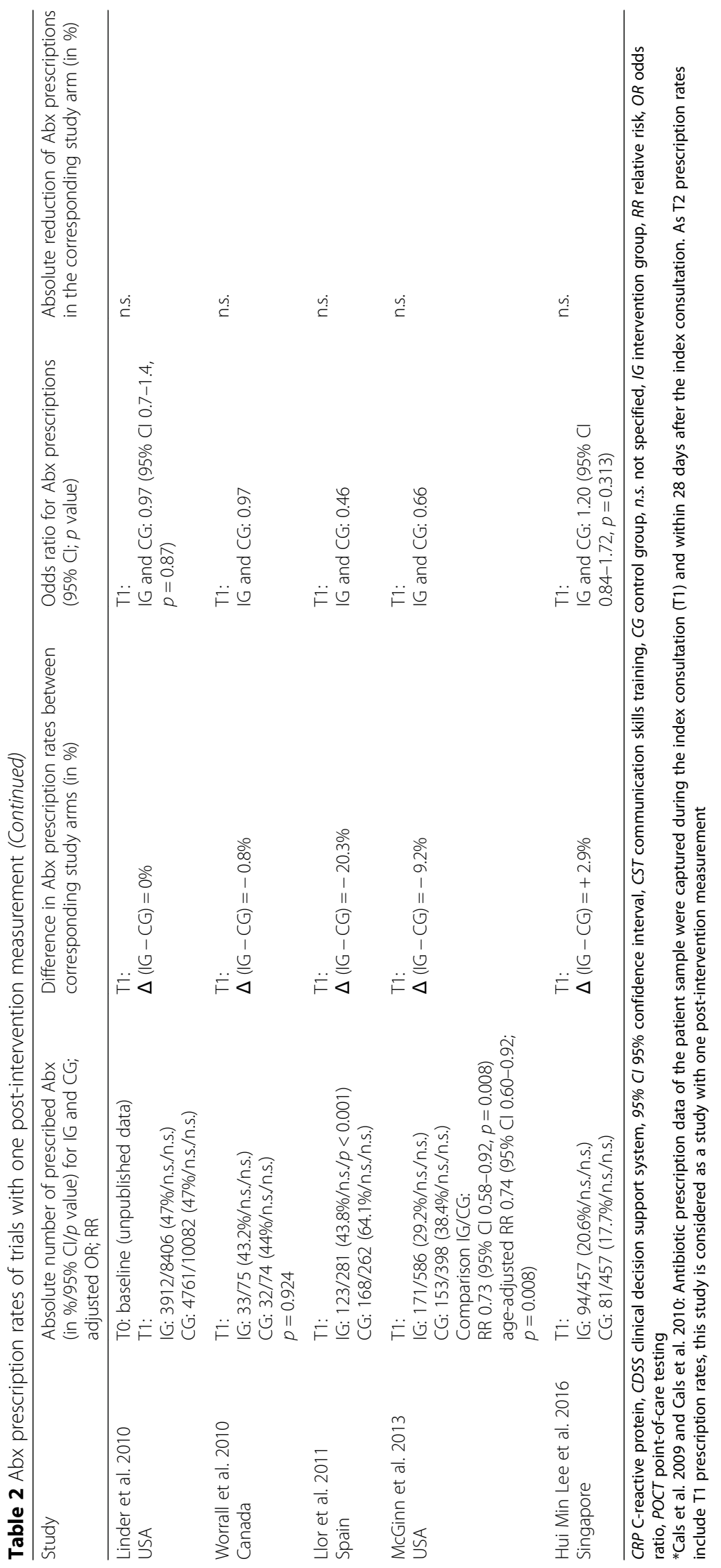




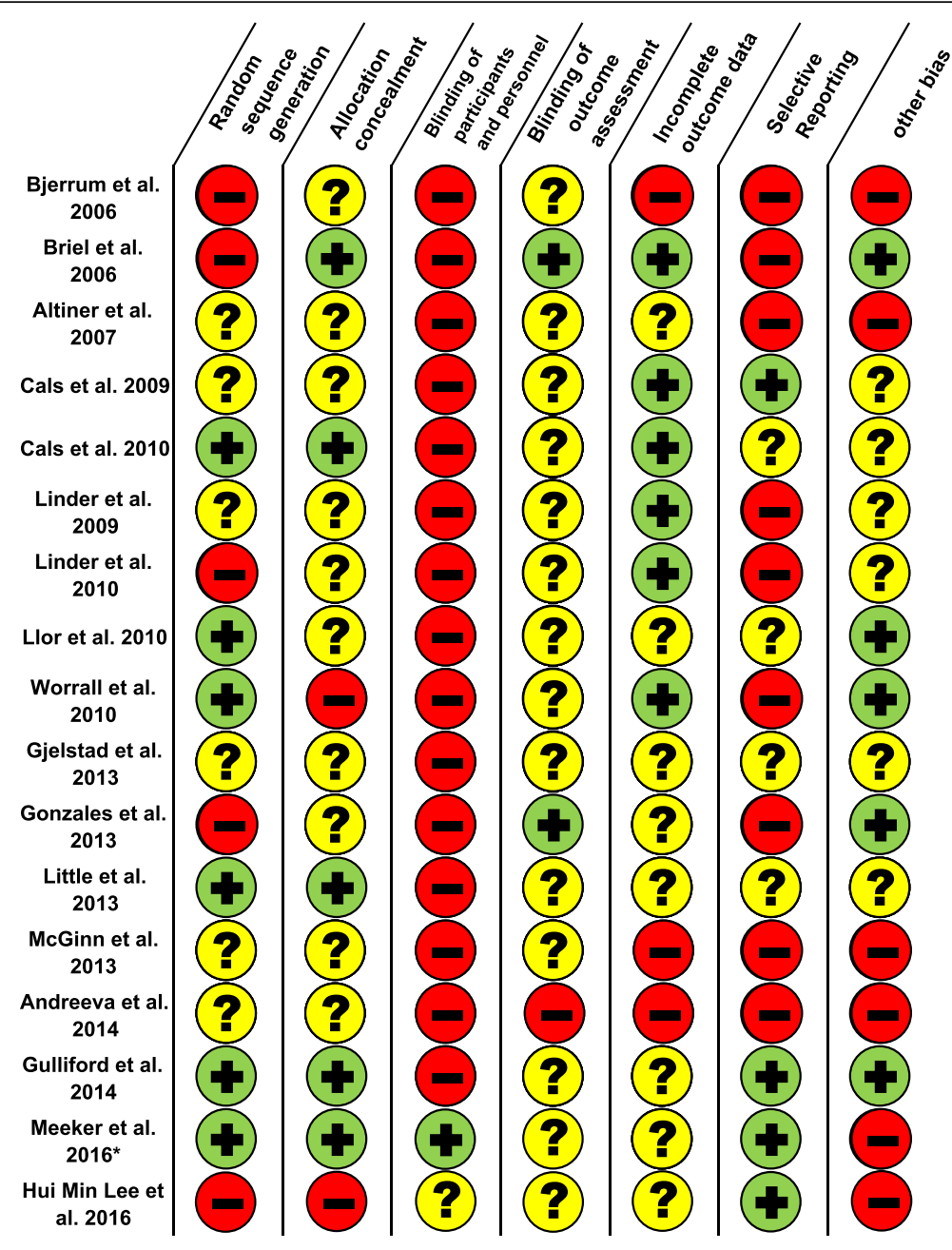

Fig. 1 Assessment of risk of bias. Legend: *plus data from published study protocol.

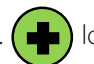

low risk of bias.

high risk of bias.

faxed or mailed prescriptions [21, 32], by field researchers [33] or by electronic medical records [13, 15, $22-25,28,29]$. Four trials used special documentation software [14, 23-25].

The time period for registration of Abx prescriptions ranged from right after the initial consultation up to 28 days after initial consultation.

Six trials assessed effectiveness of the intervention after a longer period of time, within 1 year $[15,29]$ or within 18 months after the intervention [30], after 1 year $[13,20]$ or after 3.5 years (Tables 3 and 4) [34].

\section{Description of participating physicians}

Ten trials recruited primary care physicians in private practices $[13,14,20-22,26-29,32]$, and seven trials recruited physicians from primary care clinics $[15,23-25$, 30, 31, 33].

\section{Description of patient population}

Patients $\geq 13$ years with acute upper and lower RTIs were included. The average age of patients was similar across trials and ranged from 40 to 53 years. The number of registered consultations varied from 149 to $1,115,359[28,32]$.

\section{Description of interventions Multifaceted interventions}

Twelve RCTs used multifaceted interventions [13, 14, 20 26, 28-30]. Multifaceted interventions contain two or more components and address the different aspects of inadequate antibiotic prescribing. Due to the multifaceted interventions of the included trials, some of them are discussed repeatedly in the following subsections. For example, the factorial study design of Cals et al. allows reporting of the effect of the CST or POCT alone or combined [22]. 


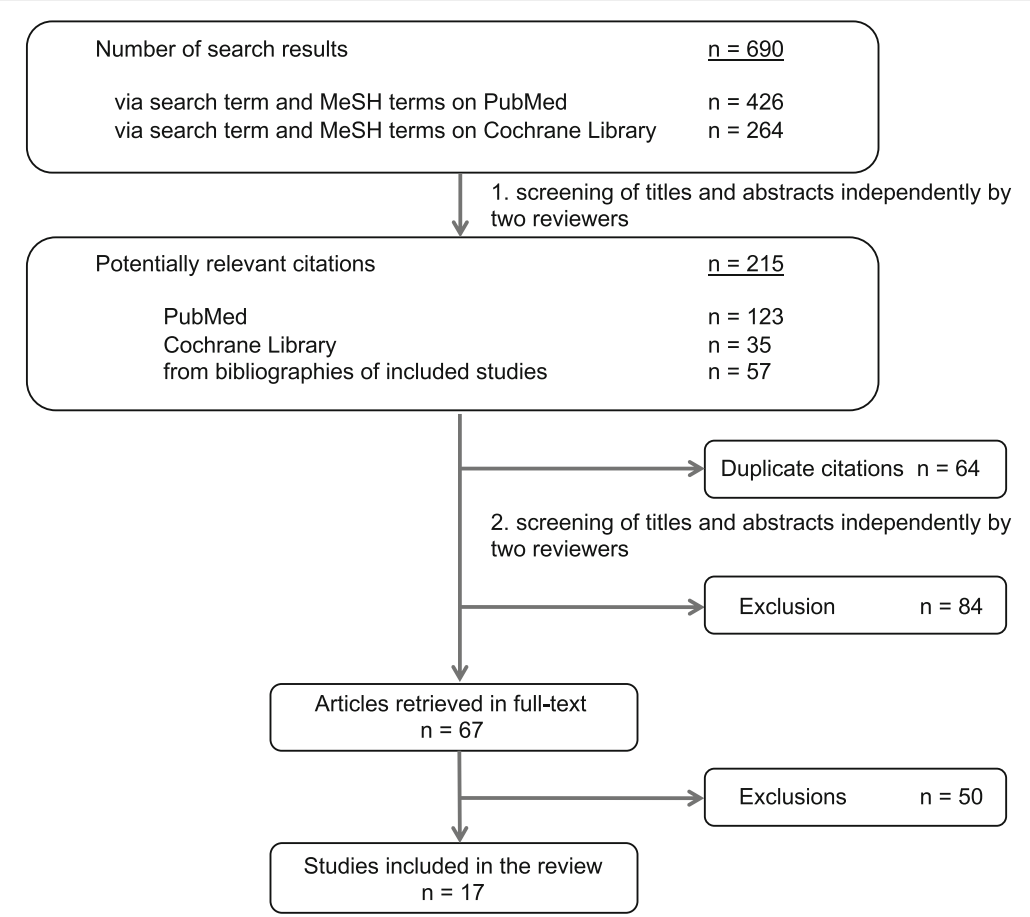

Fig. 2 Process of trial selection. MeSH - medical subject heading

\section{Intervention elements addressing physicians}

Twelve trials used interventions that addressed physicians $[13,14,20-24,26,28-30,32]$. Four different types of interventions were evaluated:

1. A "classic" knowledge transfer approach using interactive seminars $[13,14,21]$ and distribution of printed teaching and information materials [21, 23] as well as feedback on individual Abx prescription rates [13, 14, 21, 22, 24, 29]. Themes discussed included diagnosis-making and therapy of RTIs in accordance with guidelines as well as the challenge of increasing bacterial resistance.

2. A CST dealing with perceived pressure to prescribe where physicians learnt how to communicate with patients about their expectations on antibiotic prescribing and how to respond to patients' concerns. In three trials, physicians were trained in seminars [20-22]. One trial provided an Internet-based CST [26].

3. Physicians were introduced to the concept of DP. This implies advising patients with low probability of bacterial RTI to use a prescription for antibiotics only in case symptoms do not resolve or get worse up to a pre-defined point in time. Cals et al. combined this strategy with C-reactive protein (CRP) POCT [30]. POCT are simple diagnostic tests and allow measuring CRP directly in the practice. CRP is an acute-phase protein with increasing plasma concentration during inflammatory processes. The measurement of CRP with a POCT has been proved accurate and can increase diagnostic certainty if combined with clinical examination-especially for identifying patients at high risk of pneumonia [35, 36]. Gjelstad et al. implemented additional software applications that asked physicians to specify whether the concept of DP was used and to document the number of days agreed to postpone antibiotic use [14]. Worrall et al. compared two DP procedures-one of them employing a ready-to-use prescription and the other applying a post-dated prescription usable only up to $48 \mathrm{~h}$ after initial consultation [32]. Gulliford implemented information about DP within a CDSS [28].

4. Electronic health records (EHR) asked physicians to justify their treatment decision if an antibiotic was ordered and provided alternative treatment interventions [29].

\section{Intervention elements addressing patients}

Five trials implemented interventions addressing patients [20, 23, 25, 28, 33]. Four trials used patient brochures with information about RTIs as adjunct [20, $23,25,28]$. One trial used an additional waiting room poster addressing increasing bacterial resistance and prescribing pressure as one of the main reasons for inadequate prescribing [20]. Linder et al. and Gulliford et al. implemented documentation software with the possibility to print patient information leaflets $[25,28]$. In the RCT of Linder et al., it remained unclear how 


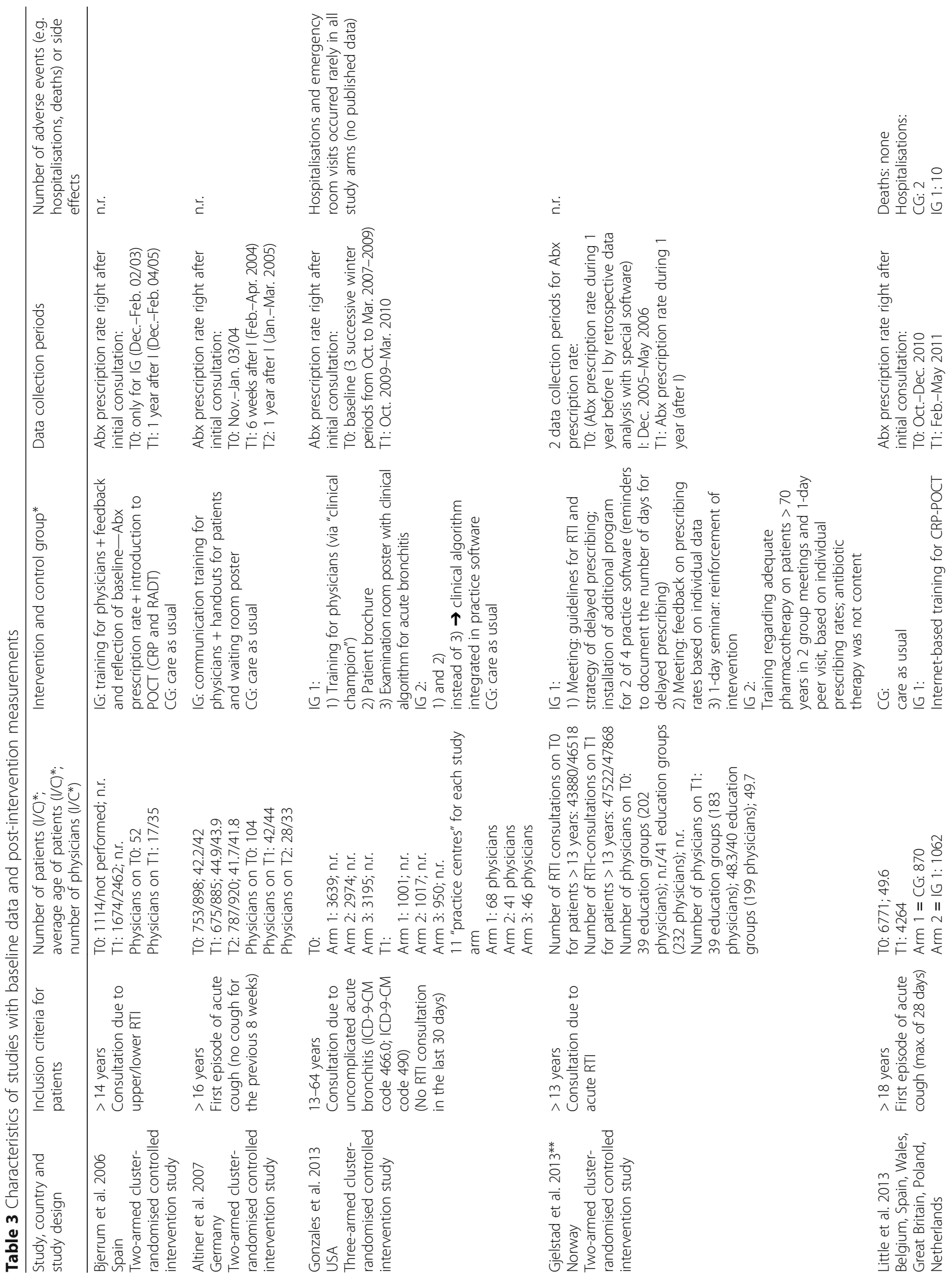




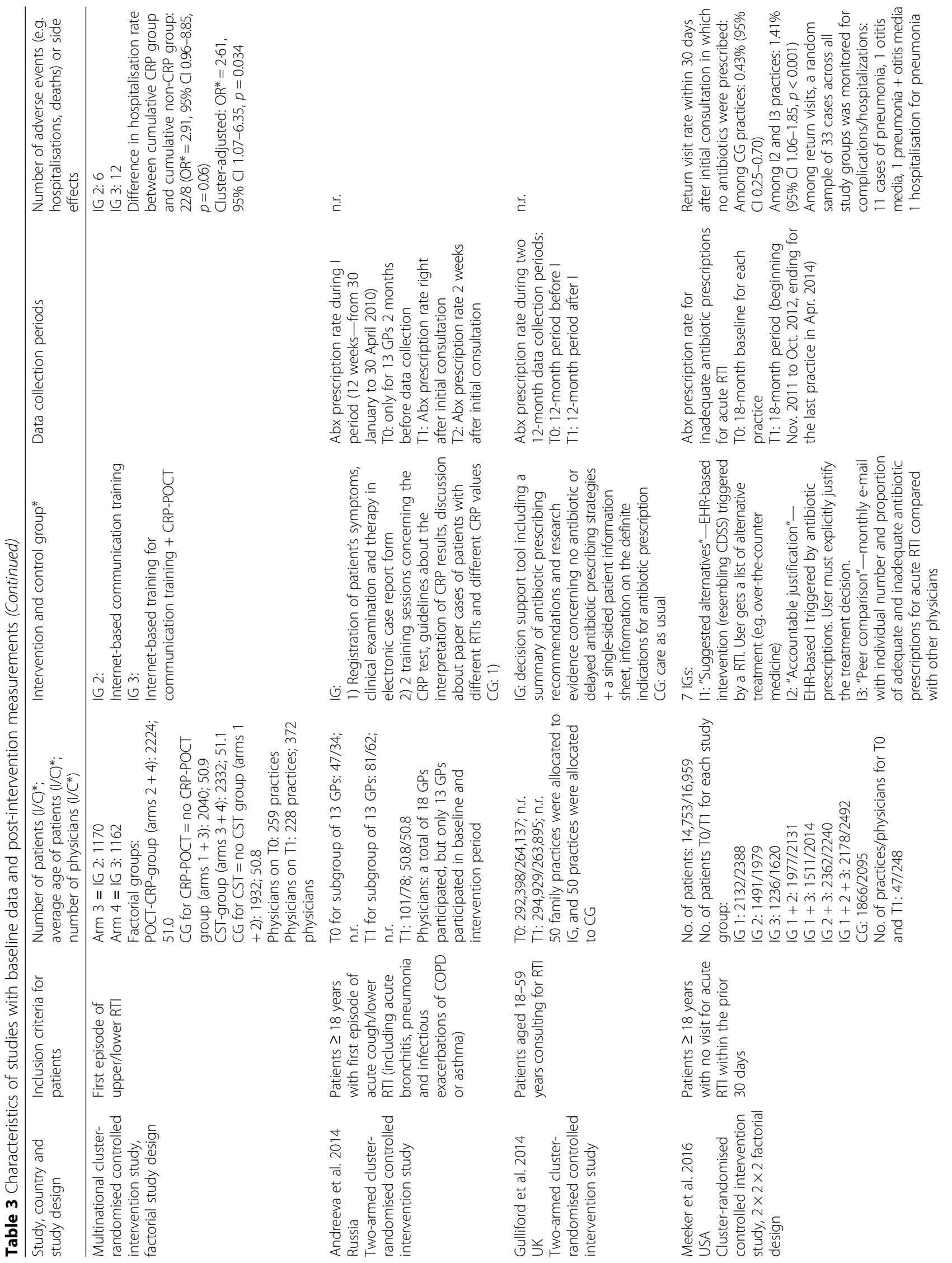




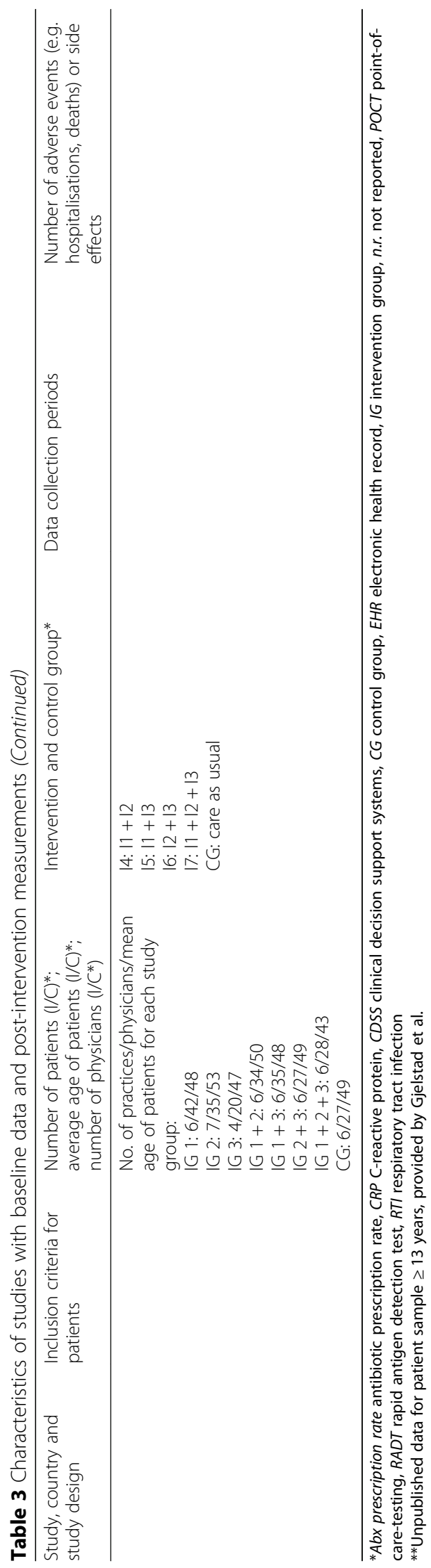




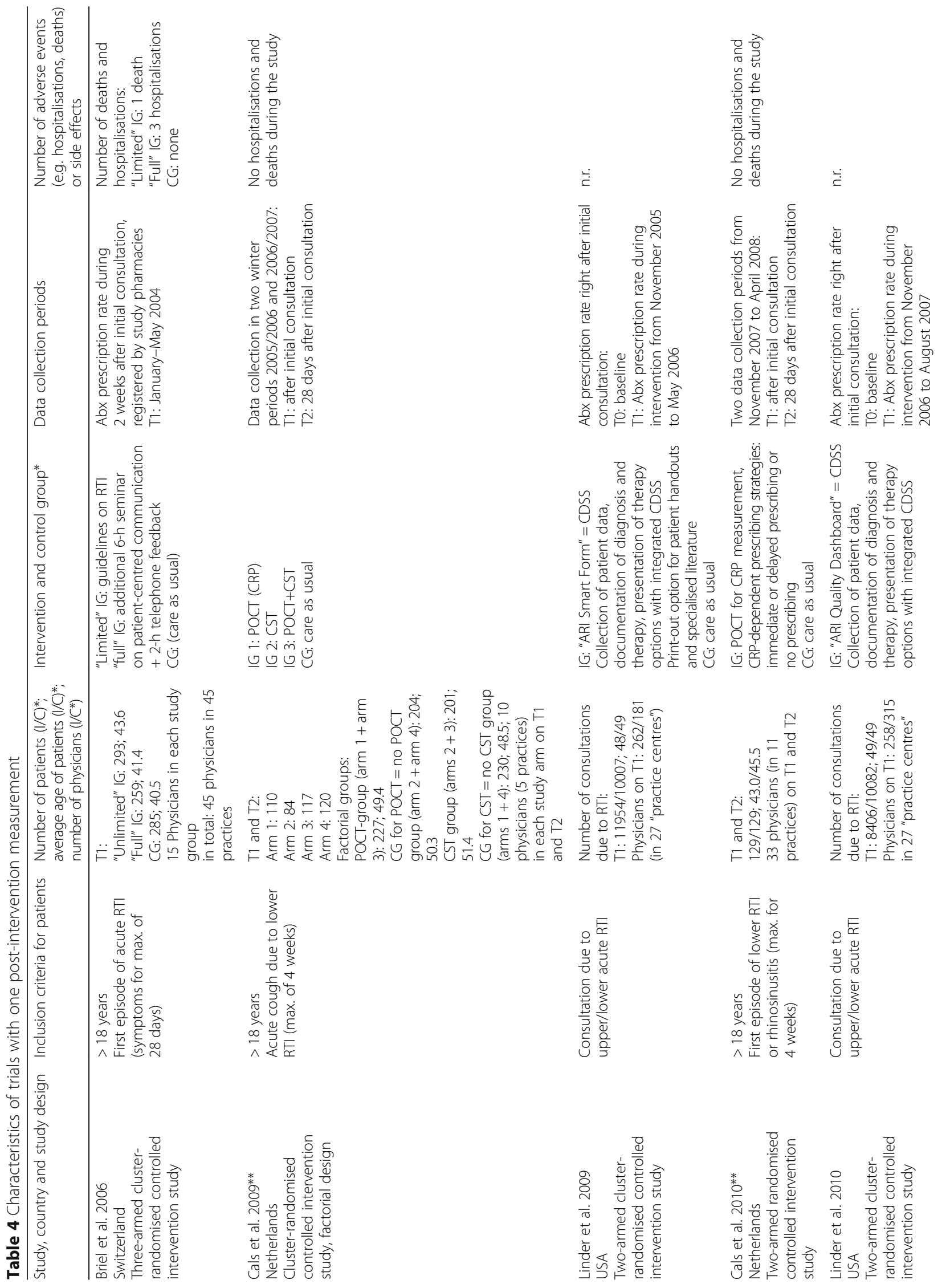




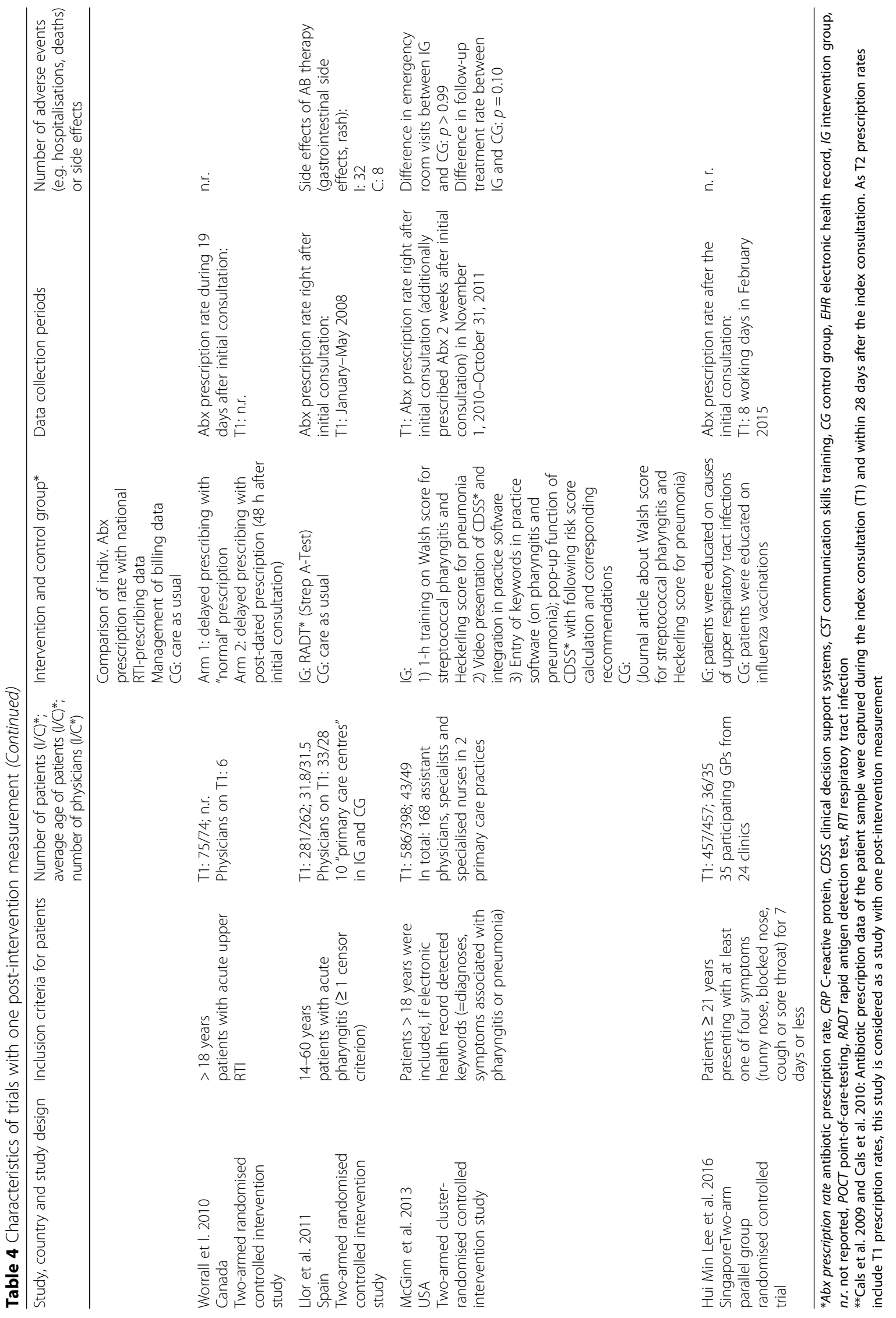


many physicians used this possibility [25]. In the trial of Gulliford et al., the number of printed leaflets was low among the physicians with the highest utilisation of the CDSS (25 leaflets per 1000 consultations for RTI) [28]. In the trial of Hui Min Lee et al., patients in the IG were educated on the aetiology of upper RTIs by trained field researchers prior to the consultation [33].

Intervention elements addressing improved diagnosis-making Twelve trials implemented interventions addressing diagnosis-making [13, 15, 22-31]. In one trial, the POCT was combined with a CST [22]. Another trial combined POCT with the strategy of DP [30]. Little et al. provided CRP-POCT training via the Internet [26]. Andreeva et al. used the CRP-POCT as a single intervention [27]. Bjerrum et al. employed a rapid antigen detection test for identifying group A streptococcal infections (RADT) in combination with feedback on personal prescribing rates [13]. Llor et al. used the RADT by itself [31]. The RADT is a fast pathogen identification test and can assist a physician in differentiating between a bacterial pharyngitis caused by group A streptococci or a viral infection. In combination with clinical scores such as the McIsaac score, it can raise diagnostic certainty and help to avoid unnecessary antibiotic prescriptions [37].

Six trials made use of CDSS [15, 23-25, 28, 29]. Linder et al. [24, 25] and McGinn et al. [15] provided assistance for estimating the likelihood of a bacterial RTI [24, 25] or a pneumonia/streptococcal pharyngitis [15]. Gulliford et al. provided evidence from research for antibiotic prescribing when a RTI was coded in an electronic medical record [28]. Andreeva et al. compared two different methods of diagnostic assistance: a computer-based system and a poster with a clinical algorithm [23]. Meeker et al. asked their participating physicians to justify their entered diagnosis and treatment [29].

\section{Effects of the intervention on Abx prescription rate (see Tables 1 and 2 and Figs. 3 and 4 )}

Twelve trials reported statistically significant lowered Abx prescription rates in the IGs compared to CGs [13-15, 20, 22, 23, 26-31]. In five RCTs. the Abx prescription rates could not be reduced significantly (see Tables 1 and 2) [21, $24,25,32,33]$. Using our definition for a clinically relevant reduction of prescription as criterion for efficacy, only six trials had a meaningful effect on Abx prescription rates [22, $23,26,27,30,31]$. The effect of the interventions cannot be compared directly due to heterogeneous study designs.

\section{Intervention effect of trials with baseline and post-intervention measurements}

The Abx prescription rates of studies with baseline and post-intervention measurements are provided in Fig. 3.
In the trial of Bjerrum et al., the CG has in contrast to the IG no pre-intervention measurement [13]. All but one had an observation period ranging from measurement direct after the consultation up to 1 year [27]. The baseline prescription rates ranged from 24.4\% [29] to $80 \%$ [23]. All studies found statistically significant results within and/or in-between study arms. The trial of Altiner et al. showed a large difference in baseline prescription rates, indicating the possibility of selection bias. Additionally, the initially observed effects after 6 weeks were not sustainable in this trial (see Fig. 3) [20]. Three studies reached a clinically relevant difference in differences greater than $10 \%$ [23, 26, 27]. Although, Gjelstad et al. observed a statistically significant reduction in prescribing rates, the overall effect of $-1.52 \%$ in the IG compared to $+1.7 \%$ in the CG is negligible [14] . Comparing baseline and post-intervention Abx prescription rates, changes within the IGs ranged from +0.3 to $-23.3 \%[20,26]$. Changes within CGs ranged from + 10.1 to $-13.1 \%[20,29]$.

\section{Intervention effect of trials with post-intervention measurements}

A total of nine studies reported on post-intervention Abx prescription rates (Fig. 4) [15, 21, 22, 24, 25, 30-33].

The reported prescription rates ranged from $13.5 \%$ [21] to $67 \%$ [22]. Only four of those trials observed a statistically significant reduction of Abx prescription rates $[15,22,30,31]$, but only three of them reached a difference between IG and CG exceeding 10\% [22, 30, 31].

All three studies with a factorial design had a positive effect on reducing Abx prescription rates [26, 29, 30]. Studies with reported prescription rates lower than $20 \%$ had no significant reduction in Abx prescribing rates [21, 33].

Differences of $+2.9 \%$ to $-44 \%$ were observed in the IGs compared to CGs on post-intervention periods [22, 33] (see Table 2).

\section{Effects of single-element interventions}

Single-element interventions address one specific reason for inadequate prescribing. Nine RCTs implemented singleelement interventions [15, 21, 22, 26, 27, 29, 31-33], three of them within a factorial study design [22, 26, 29].

All these RCTs contained interventions addressing either physicians [21, 22, 26, 29, 32], patients [33] or diagnosis-making $[15,22,26,27,29,31]$. In the RCT by Worrall et al., DP with a post-dated prescription did not significantly reduce antibiotic use compared to usual DP [32]. Hui Min Lee et al. did not find significantly reduced Abx prescriptions by means of patient education on causes of upper RTIs compared to CG (20.6 vs. $17.7 \%$ in the CG, OR $=1.20, p=0.313$ ) [33]. The limited intervention in the RCT by Briel et al. could not significantly reduce Abx prescriptions [21]. 


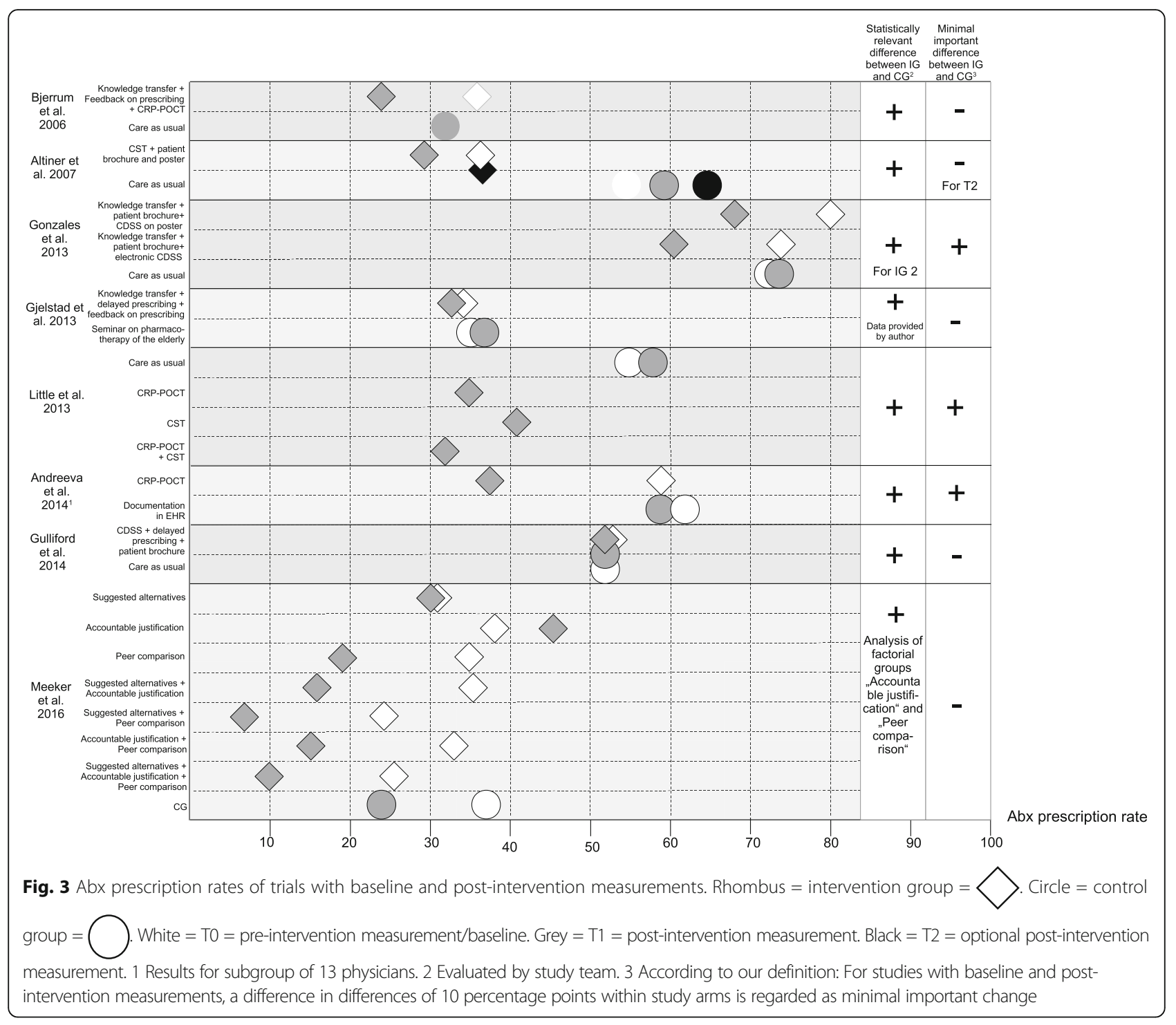

Llor et al. implemented a more effective intervention: After initial consultation, the Abx prescription rate was $44 \%$ in the IG $(p<0.001)$ as compared to $64 \%$ in the CG due to RADTs [31].

McGinn et al. observed an Abx prescription rate of 29\% in the IG and a rate of $38 \%$ in the CG $(p=0.008)$. Andreeva et al. registered a significant difference in antibiotic prescriptions within 2 weeks after initial consultation: $40.6 \%$ in the IG and $71.8 \%$ in the CG $(p=0.0001)$ [15].

In the trial by Cals et al., CRP-POCT reduced the Abx prescription rate by $13 \%$ compared to control $(p<0.01)$ [22]. Investigating long-term effects, after 3.5 years no effect was found. CST was able to reduce the $\mathrm{Abx}$ prescription rate, re-measured 28 days after consultation, by $25 \%(p<0.001)$. After 3.5 years, patients in the CST group received significantly less antibiotic prescriptions for RTIs than in the CG $(p<0.02)$ [34].
Little et al. reduced the Abx prescription rate in the cumulative CST group ( 36 vs. $45 \%$, OR $=0.69 ; 95 \%$ CI 0.54-0.87, $p<0.0001$ ) and CRP POCT group (33 vs. $48 \%$; OR $=0.54 ; 95 \%$ CI $0.42-0.69, p<0.0001)$ compared in the control [26].

Meeker et al. implemented three single-element interventions (two of them focusing at physicians, one focusing at diagnosis-making) in a factorial study design: Intervention 1 "Suggested alternatives" did not significantly reduce Abx prescriptions ( $p=0.66$ for differences in trajectories). Intervention 2 "Accountable Justification" and intervention 3 "Peer comparison" registered significant differences in the rate of inappropriate antibiotic prescribing $(p<0.001)$ [29].

\section{Effects of multifaceted interventions}

Multifaceted interventions address different aspects of inadequate antibiotic prescribing-such as CST or 


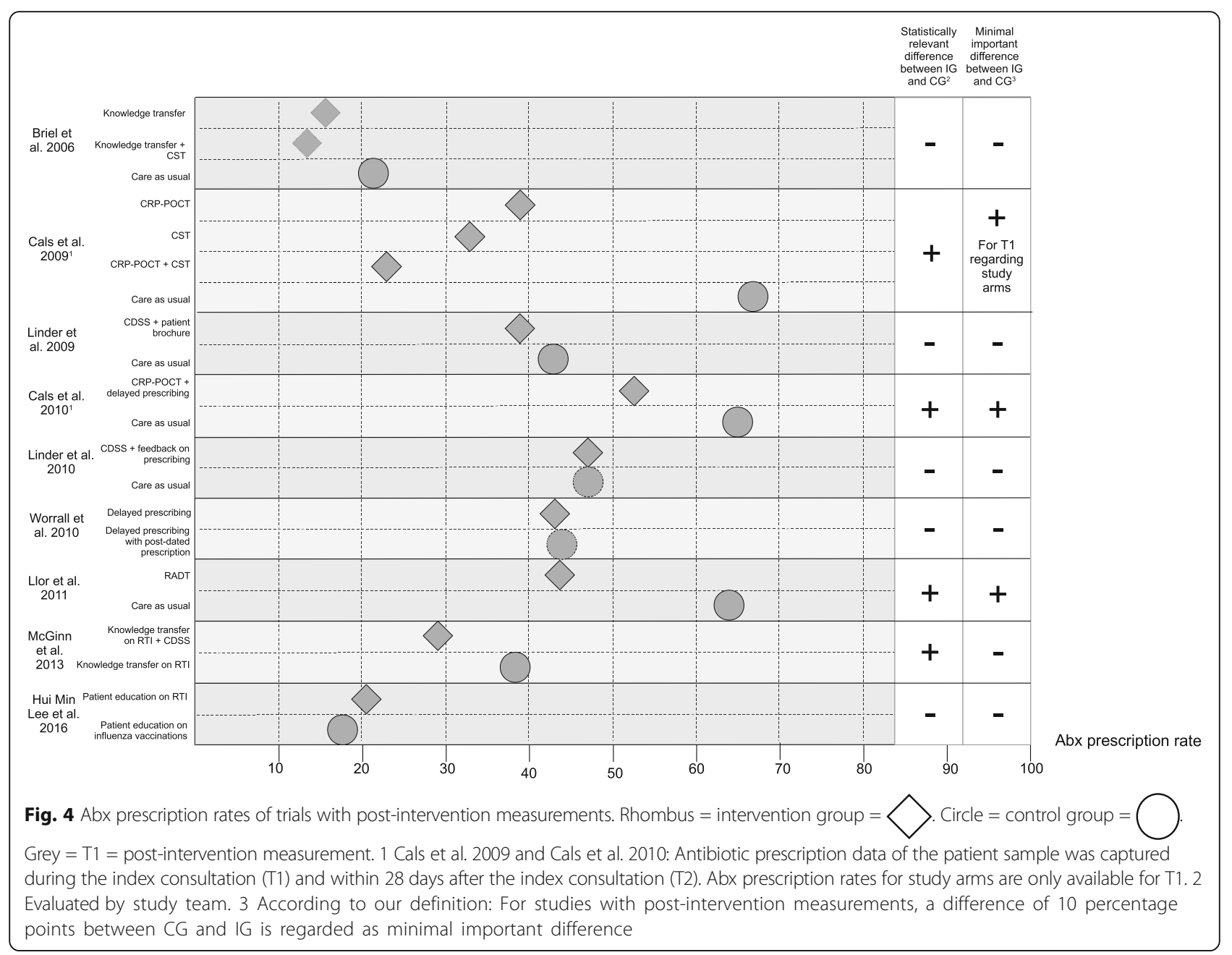

POCT addressing prescribing pressure or diagnostic uncertainty. In most cases, multifaceted interventions contain intervention elements from at least two out of three "target groups": physicians, patients or the process of diagnosis-making [13, 20-26, 28-30]. However, according to our definition, a multifaceted intervention can only focus at physicians or patients or diagnosismaking if different aspects are taken into account [14]: One RCT can just focus at physicians but with prescribing feedback and implementation of DP serving as a multifaceted intervention.

Interventions focusing on patients alone were mostly used as additional elements in multifaceted interventions $[20,23,25,28]$.

\section{Effects of multifaceted interventions addressing} physicians, patients and the process of diagnosis-making Two RCTs implemented interventions addressing all three "target groups". Both trials could significantly reduce their Abx prescription rates [23, 28]. Gulliford et al. combined a CDSS with patient handouts and DP.
The proportion of consultations with antibiotics prescribed declined marginally from 53 to $52 \%$ during 12 months after intervention, whereas it remained constant at $52 \%$ in the CG. The adjusted mean difference in antibiotic prescriptions was $-1.85 \%(p=0.038)$ [28]. Gonzales et al. observed a reduction of $12 \%$ in the first IG (poster with clinical examination algorithm) and a reduction of $13 \%$ in the second group (CDSS). Both interventions were combined with patient handouts, feedback on prescribing and seminars for physicians. Compared to CG, both interventions were statistically significant $(p=0.003$ or $p=0.01)$, but not between themselves $(p=0.67)[23]$.

\section{Effects of multifaceted interventions addressing physicians and the process of diagnosis-making}

Five RCTs combined physician- and diagnosis-centred interventions [13,22, 24, 26, 30], four of them reducing Abx prescription rates to a statistically significant extent [13, 22, 26, 30]: In their 2010 trial, Cals et al. reduced the Abx prescription rate by $12 \%$ ( $R R$ in the $\mathrm{IG}=0.81$, 
95\% CI 0.62-0.99) within a 28-day follow-up with the help of CRP POCT and DP [30]. Bjerrum et al. implemented CRP POCT, seminars for physicians and feedback on prescribing. The Abx prescription rate in the IG was $24 \%$ (CG 32\%) 1 year after the intervention [13]. In the RCT by Little et al., the combination of CST and CRP-POCT led to a significant reduction of Abx prescriptions compared to CG (58 vs. $32 \% ; p<0.001$ ) [26]. As the trial of Cals et al. was designed as a factorial trial, there was no testing for significance for the multifaceted intervention consisting of CRP POCT and CST [22]. The Abx prescription rate at index consultation was $23 \%$ (95\% CI 11.6-34.6) and lower than in the CG (67\%; 95\% CI 53.9-79.5). Prescription rates for follow-up were not indicated. The RCT by Linder et al. from the year 2010 used CDSS and feedback on prescribing and found no difference between IG and CG [24].

\section{Effects of multifaceted interventions addressing patients and the process of diagnosis-making}

The RCT by Linder et al. from 2009 tested patient handouts in combination with a CDSS. This intervention led to a non-significant reduction of $4 \%$ in the IG [25].

\section{Effects of multifaceted interventions addressing patients and physicians}

In the trial by Altiner et al., the intervention contained patient brochures, a waiting room poster with information on RTI and a CST for physicians. The observed Abx prescription rate after 1 year was $36.7 \%$ in the IG compared to $64.8 \%$ in the CG. Yet, different baseline rates should be considered (36.4 vs. 54.7\%) [20].

\section{Effects of multifaceted interventions focusing at physicians}

Three RCTs used multifaceted interventions focusing at physicians [14, 21, 29]. Gjelstad et al. observed a reduction of $1.5 \%$ in the IG ( $p$ value $=0.027$ ) [14]. The IG received feedback on prescribing, DP and seminars for physicians. However, they registered an increase of $1.7 \%$ in the CG ( $p$ value $=0.002$ ). Continuing medical education groups with the corresponding prescription rates served as calculation basis instead of physicians with their individual prescribing rates.

In the trial of Briel et al., physician education alone reduced Abx prescription rates by $5.7 \%$. In combination with CST, the reduction was increased to $7.9 \%$ compared to the CG. Both differences were not statistically significant [21]. The combination of interventions in the RCT by Meeker et al. did not result in statistically significant lowered inappropriate Abx prescription rates compared to CG [29].
Relevance of intervention effects (see Table 6)

Table 6 shows the number of studies and intervention concepts with clinically relevant reductions of Abx prescription rates with regard to type of intervention. Six trials had a meaningful effect on Abx prescription rates [22, 23, 26, 27, 30, 31]. Three out of these six RCTs had a three-armed or factorial study design, therefore containing more than one intervention concept $[22,23,26]$. Altogether, our review contains 11 clinically relevant intervention concepts. The majority contained diagnosiscentred elements $[22,23,26,27,30,31]$, especially CRP POCT [22, 26, 27, 30].

POCT (CRP and RADT) and CST reduce effectively antibiotic prescriptions alone or in combination [22, 23, $26,30]$. One RCT combined CDSS with knowledge transfer and patient handouts effectively [23].

\section{Clinically relevant single-element interventions}

Six single-element intervention concepts could reduce Abx prescription to a clinically relevant extent [22, 26, $27,31]$. Four out of these six single-element interventions used diagnosis-centred interventions [26, 27, 31, 34]. All four tested POCT: Three measured the level of CRP [22, 26, 27], and one RCT used a RADT [31].

Two RCTs implemented the CST as an intervention focusing at physicians $[22,26]$.

\section{Clinically relevant multifaceted interventions}

Five intervention concepts led to a clinically relevant reduction $[22,23,26,30]$. All interventions combined elements focusing at physicians and at the process of diagnosis-making $[22,26,30]$. Two intervention concepts had additional patient-centred elements [23]. The diagnosis-centred interventions contained CRP POCT $[22,26,30]$ and CDSS [23].

\section{Secondary endpoints}

Predefined secondary endpoints were patient-centred outcomes (e.g. reconsultation rate [21-23, 25-27, 29], patient satisfaction [21, 22, 30], patients' views on RTIs [33] or days to recovery [22, 26, 27, 30, 31]), outcomes related to antibiotic prescribing (e.g. Abx prescriptions according to guidelines [24, 25, 31], class of prescribed antibiotic [13-15, 31], prescribed antibiotics for specific RTIs [13, 28]) or diagnostics (e. g. number of X-rays [27], number of RADTs [15]). Four RCTs did not find any significant difference in secondary endpoints between IG and CG [20-23]. One RCT did not provide any information on secondary endpoints [32].

\section{Differences in patient-centred secondary outcomes}

Four trials reported on differences in patient-centred secondary outcomes $[26,29,30,33]$. Patient satisfaction was higher in the IG (76.3 vs. $63.2 \%$ of patients who 
were at least very satisfied, $p=0.03$ ) [30]. Patient's understanding of RTI improved $(p<0.001)$ [33], and the return visit rate within 30 days after a visit without antibiotic prescription was higher in the IG (1.41 vs. $0.43 \%$ in the CG, $p<0.001$ ) [29]. Patients treated by physicians who had been trained in CST required more days for resolution of symptoms in the IG (adjusted risk ratio in the IG $0.83, p=0.002)$ [26].

\section{Differences in physician-centred secondary outcomes}

The Abx prescription rate for patients with specific RTIs such as acute cough and bronchitis was significantly lower in the IG in the trial by Gulliford et al. $(-2.49 \%$, 95\% CI $0.15-4.83 \%, p=0.030$ ) [28]. Four RCTs reported on prescribed classes of antibiotics [13-15, 31] (see Table 5-intervention influence on prescribed antibiotic class). The trial by Llor et al. is omitted in this table because it did not distinguish between IG and CG [31]. Two of these four trials witnessed a significant increase of narrow-spectrum penicillins [13, 14], whereas the proportion of other antibiotic classes declined: In the trial by Gjelstad et al., the prescribing of tetracylines and macrolides decreased significantly in the IG. Simultaneously, the proportion of macrolides increased in the CG [14]. McGinn et al. observed significant differences only for the prescription rates for quinolones. The prescribing rate in the IG was $9.9 \%$ compared to $19.6 \%$ in the CG $(p=0.02)$ [15].

Four RCTs categorised the prescribed antibiotics in appropriate or inappropriate prescriptions $[24,25,31]$. However, definitions for (in-) appropriate prescribing differed: Linder et al. and Meeker et al. defined antibiotic-appropriate (pneumonia, streptococcal pharyngitis, sinusitis, otitis media) and non-antibioticappropriate diagnoses (non-streptococcal pharyngitis, influenza, acute bronchitis, non-specific upper RTIs) [24, 25]. Llor et al. defined antibiotic prescribing for patients without group A streptococcal infections as well as missing antibiotic treatment for patients with group A streptococcal infections as inappropriate [31].

Linder et al. implemented a CDSS. When a CDSS was used, the rate of antibiotic prescriptions for diagnoses with appropriate antibiotic treatment was higher as in consultations without CDSS (88 vs. $59 \%$, OR $=5.0$; $95 \% \mathrm{CI}=2.9$ 8.6) [25]. The rate of non-appropriate antibiotics was lower when physicians used the CDSS ( 32 vs. $43 \%, p=0.004$ ) [24]. Physicians who made use of a CDSS prescribed less antibiotics in all consultations for RTI ( 42 vs. $50 \%, p=0.02$ ) [24]. Meeker et al. found reductions from 15.6 to $19.5 \%$ in seven IGs due to behavioural interventions alone or in combination [29] (see Table 1 for details).

In the trial by Llor et al., a RADT test was tested. A higher number of inappropriate antibiotics was observed in the CG (60 vs. $27 \%$ in the IG, $p<0.001)$ [31].

\section{Differences in diagnosis-centred secondary outcomes}

The RCT by Andreeva et al. observed a lower rate for chest radiography in the IG (55.4 vs. $76 \%, p=0.004)$ [27].

\section{Discussion}

\section{Summary of main results}

This review updates and summarises current evidence of various interventions in primary care on reducing $A b x$ prescriptions in patients $\geq 13$ years for acute RTI. Twelve out of 17 included RCTs showed a statistically significant lower Abx prescription rate in the IG [13-15, $20,22,23,26-31]$. However, only six of them reported a clinically relevant reduction according to our definition $[22,23,26,27,30,31]$. Due to the three-armed or factorial study design, these six RCTs contained 11 clinically relevant intervention concepts. Interventions focusing at physicians (CST) and at the process of diagnosis-making (CRP POCT, RADT, CDSS) were-alone or in combination-the most effective interventions. Observed reductions for RCTs with baseline ranged from 1.5 to $23.3 \%$ and cannot be compared directly due to heterogeneous baseline Abx prescription rates, study designs and settings $[14,26]$. For studies with post-intervention measurements, the differences between IG and CG were between 2.9 and $-44 \%$ [22, 33]. Studies with reported prescription rates below 20\% did not show significant reductions in Abx prescribing rates [21, 33]. Postintervention observation periods ranged from 2 weeks up to 3.5 years. Conclusions on long-term sustainability of interventions cannot be drawn.

\section{Meaning of the results and comparison with existing literature}

Our findings are in line with other systematic reviews, which reported mixed results regarding interventions to reduce antibiotics with either a larger or narrower spectrum of interventions and setting $[7,8,10,38]$. We focused on RTIs in primary care excluding other infectious conditions and settings like emergency rooms, hospitals or public campaigns $[39,40]$.

We included RCTs with single-element $[15,22,26,27$, $29,31-33]$ and multifaceted interventions [13, 14, 20$26,29,30]$ focusing at diagnosis-making [13, 15, 22-31], at physicians $[13,14,20-24,26,28-30,32]$ or at patients $[20,23,25,28,33]$. Nine intervention concepts with meaningful effects on Abx prescription rates contained interventions addressing the process of diagnosismaking $[22,23,26,27,30,31]$, five of them in combination with interventions targeting physicians $[22,23,26$, $30]$. In contrast to the systematic review of 2005, singleelement interventions can be effective (Table 6) [22, 23, 26, 27, 30, 31]. Interventions addressing patients were less likely to reduce Abx prescriptions to a meaningful extent. POCT (CRP and RADT) and CST-alone or in 


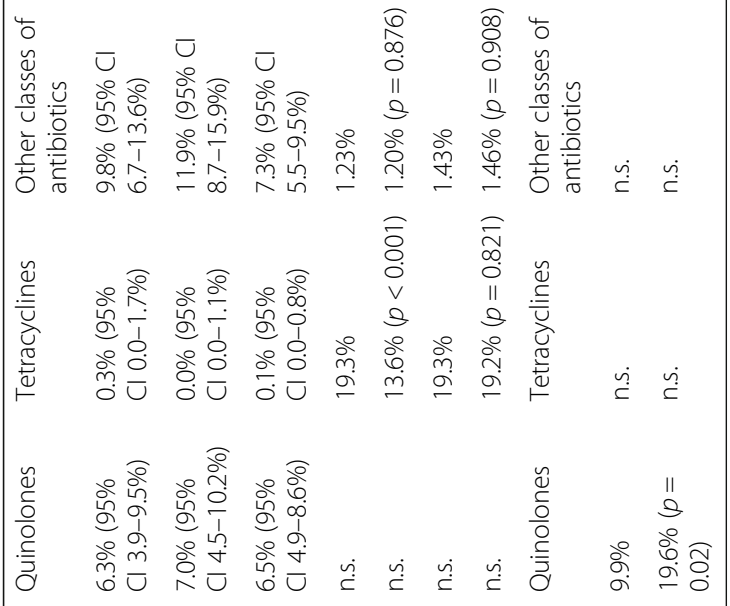

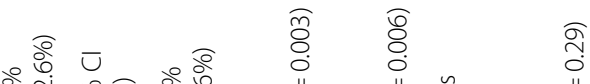

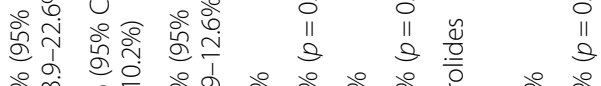

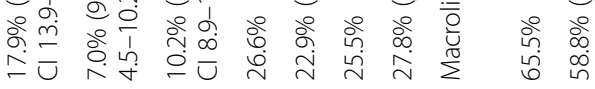
类

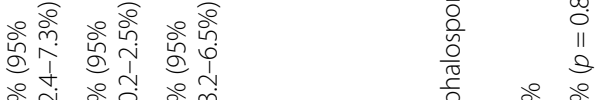

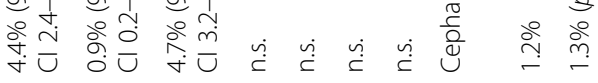

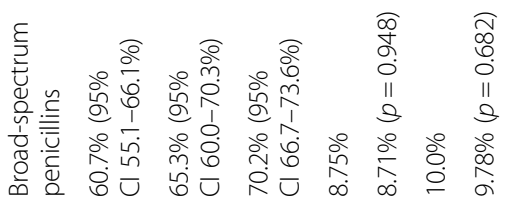

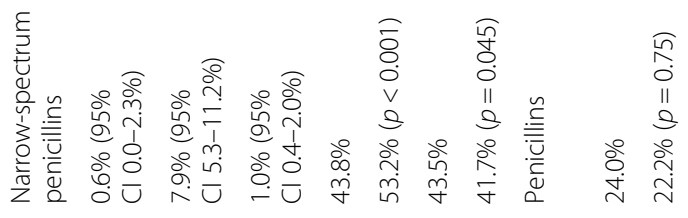

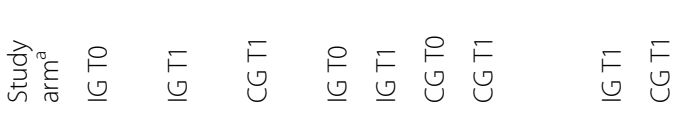

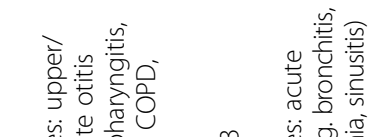


Table 6 Clinically relevant interventions

\begin{tabular}{|c|c|c|c|c|c|c|}
\hline Study & $\begin{array}{l}\text { Multifaceted } \\
\text { intervention }\end{array}$ & $\begin{array}{l}\text { Single-element } \\
\text { intervention }\end{array}$ & $\begin{array}{l}\text { Intervention } \\
\text { focusing at } \\
\text { diagnosis-making }\end{array}$ & $\begin{array}{l}\text { Intervention } \\
\text { focusing } \\
\text { at physicians }\end{array}$ & $\begin{array}{l}\text { Intervention } \\
\text { focusing } \\
\text { at patients }\end{array}$ & $\begin{array}{l}\text { Details about the } \\
\text { intervention }\end{array}$ \\
\hline Cals et al. 2009 & $x$ & & $x$ & $x$ & & $\mathrm{CST}+\mathrm{CRP} P O C T$ \\
\hline Cals et al. 2009 & & $x$ & & $x$ & & CST \\
\hline Cals et al. 2009 & & $x$ & $x$ & & & CRP POCT \\
\hline Cals et al. 2010 & $x$ & & $x$ & $x$ & & CRP POCT + DP \\
\hline Llor et al. 2011 & & $x$ & & & & RADT \\
\hline Gonzales et al. 2013 & $x$ & & $x$ & $x$ & $x$ & $\begin{array}{l}\text { Software for CDSS + knowledge } \\
\text { transfer }+ \text { feedback on prescribing } \\
+ \text { patient handouts }\end{array}$ \\
\hline Gonzales et al. 2013 & $x$ & & $x$ & $x$ & $x$ & $\begin{array}{l}\text { CDSS }+ \text { knowledge transfer }+ \\
\text { feedback on prescribing }+ \\
\text { patient handouts }\end{array}$ \\
\hline Little et al. 2013 & $x$ & & $x$ & $x$ & & $\mathrm{CST}+\mathrm{CRP} P \mathrm{POCT}$ \\
\hline Little et al. 2013 & & $x$ & & $x$ & & CST \\
\hline Little et al. 2013 & & $x$ & $x$ & & & CRP POCT \\
\hline Andreeva et al. 2014 & & $x$ & $x$ & & & CRP POCT \\
\hline
\end{tabular}

CDSS clinical decision support system, CRP C-reactive protein, CST communication skills training, DP delayed prescribing, POCT point-of-care test, RADT rapid antigen detection test for group A streptococci

combination-reduce effectively antibiotic prescriptions $[22,23,26,27,30]$.

\section{Differences in Abx prescription rates and effect sizes}

We observed large differences in Abx prescription rates between countries ranging from 13.5 and $80 \%$ and within a country $[21,23]$. There were five trials from the USA $[15,23-25,29]$ where pre-intervention Abx prescription rates ranged from 24 to $80 \%$ [23, 29] and post-intervention Abx prescription rates varied from 29 to $47 \%[15,24,25]$. There were two studies from the Netherlands with post-intervention Abx prescription rates ranging from 23 to $65 \%$ [22, 30]. Two studies from Spain showed post-intervention Abx prescription rates between 24 and $64 \%$ [13, 31]. Pre-intervention Abx prescription rates in the trial of Bjerrum et al. were only available for the IG [13]. These large variations within and between countries limit the generalisability of the findings and indicate high possibility of selection bias and regional factors affecting Abx prescription rates.

Five included trials did not demonstrate any reduced Abx prescription rates [21, 24, 25, 32, 33]. Possible reasons for lack of success were possible selection bias in the recruitment of physicians who were already low prescribers [21,33], low intervention uptake or insufficient implementation $[24,25]$ as well as lack of power due to low number of participating physicians and patients [32]. We consider five trials reporting statistically significant results as ineffective [14, 15, 20, 28, 29]. Gulliford et al. used a CDSS in a large sample of family practices and reported a small difference of $1.85 \%$ (95\% CI - 0.1 to 3.59) [28]. IG and CG had a similar baseline prescription rate of roughly $50 \%$ reflecting overprescribing. The reported statistical significance of the small observed effect is due to the large sample size and cannot be regarded as efficient (difference in differences: $-1 \%$ ).

Altiner et al. implemented CST, patient brochures and a waiting room poster in the IG [20]. Despite a large difference in baseline prescription rates and increased $\mathrm{Abx}$ prescription rates in the IG $(+0.3 \%)$ and CG $(+10.1 \%)$ within 1 year after baseline, this trial reported statistically relevant reductions after adjusting for seasonal effects and confounding variables such as severity of disease (IG: adjusted OR $=0.72,95 \%$ CI 0.54-0.97, $p=0.028$; CG: adjusted $\mathrm{OR}=1.31,95 \% \mathrm{CI}$ $1.01-1.71, p=0.044)$. This reduction does not satisfy our conditions for a meaningful change (difference in differences $-9.8 \%$ ).

In the trial of Gjelstad et al., the combination of knowledge transfer, DP and feedback on prescribing resulted in a small difference of $-4.1 \%$ between IG and CG [14]. Due to the large sample size, this trial reported statistically relevant reductions but cannot be regarded as a meaningful change (difference in differences $+0.2 \%$ ).

Meeker et al. investigated three interventions separately and in combination within a factorial study design [29]. Although two of the interventions showed a statistically significant and impressive reduction of Abx prescriptions of $17 \%$, this has to be interpreted given that a reduction of $13 \%$ in Abx prescriptions was also observed in the CG. An explanation for a reduction without intervention was not given, beside Hawthorne effect. This observation points out the importance of an independent CG and pre- and post-intervention measurements of 
prescribing rates. Therefore, all trials shown in Fig. 4 lacking pre-intervention measurements have to be interpreted cautiously. For example, McGinn et al. reported a trial with a difference of $9 \%$ in Abx prescription rates compared to the CG, just below our arbitrary threshold for minimal significance of $10 \%$ [15]. However, for both groups, no baseline prescription rates are available.

Our review suggests that, in countries with relatively low prescription rates like Germany or the Netherlands, CST seems to be the key element for successful interventions. In contrast, interventions focusing on making a diagnosis in terms of POCT and CDSS showed relevant reductions in high-prescribing countries (e.g. Spain, USA, Russia) [13, 23, 27]. The role of electronic decision support systems remains unclear.

\section{Secondary outcomes}

This review also adds to our knowledge that interventions aiming at reducing inappropriate antibiotic prescribing can have a positive effect on the prescribing quality. In a number of trials, the number of narrowspectrum penicillins increased $[13,14]$, whereas the proportion of broad-spectrum antibiotics declined [13-15], although the main focus of the interventions was to not prescribe. Effects on patients' satisfaction were reported in three trials $[21,22,30]$. Only one trial reported a significantly higher proportion of patients satisfied with care in the IG due to CRP-POCT $(p=0.03)$.

\section{Limitations of the review}

Our systematic literature search was limited to few databases and hand search of references due to lack of access to other databases and funding. Additionally, publication bias and the possible exclusion of some foreign language trials have to be acknowledged. Although we cannot exclude that we have missed few trials, we believe this would not have changed our conclusions or allowed summary statistics given the heterogeneity of the designs and outcome measures.

\section{Limitations of trials included}

All included RCTs differed in study design, data collection and time points of measurement, trial quality and baseline prescribing rates. Reporting of trial data was often poor due to missing $p$ values, confidence intervals, absolute number of prescriptions and/or baseline data (Tables 1 and 2). Trials with high risk of bias may have led to a too positive interpretation of reported results. The heterogeneity of trial designs and outcome measurements made it impossible to pool trial data or compare effect sizes (e.g. using Cohen's $d$ ) between trials. Alternatively, we have summarised the baseline and post-intervention Abx prescription rates in Figs. 3 and 4 to illustrate the differences and heterogeneity in between trials. There is no consensus about the effect size on Abx prescription rates considered as minimal important change. Our arbitrary assumptions considering an absolute $10 \%$ change as minimal important is based on the impression gained from these figures. The majority of trials did not adjust or balance seasonal effects (winter vs. summer), possibly affecting Abx prescription rates.

\section{Conclusions and implications for research}

CST and POCT alone or in combination have the potential to reduce antibiotic prescriptions for RTIs. Electronic decision support tools showed only mixed results. Eleven out of 17 trials were not successful in reducing Abx prescription rates according to our definition of minimal important change $[13-15,20,21,24,25,28,29$, 32, 33]. However, six of them reported a statistically significant reduction [13-15, 20, 28, 29]. Trials with low initial Abx prescription rates were less likely to be successful. Despite a number of noteworthy current studies, the generated evidence remains disappointingly limited. Only moderate evidence which interventional strategies are successful and how these findings could be generalised beyond the actual setting and the observational period of the trial exist.

We conclude that there is a need to develop a consensus for designing and reporting of trials aiming to reduce inappropriate Abx prescriptions in the near future. It should address (among others) the measurement of preintervention prescribing rates, adjustment for seasonal and temporal trends, (minimal) follow-up time, data analysis and reporting.

\section{Abbreviations \\ 95\% Cl: 95\% confidence interval; Abx prescription rate: Antibiotic prescription rate; CDSS: Clinical decision support system; CG: Control group; CRP: C- \\ reactive protein; CST: Communication skills training; DP: Delayed prescribing; EHR: Electronic health record; IG: Intervention group; OR: Odds ratio; POCT: Point-of-care testing; RADT: Rapid antigen detection test; $\mathrm{RCT}$ : Randomised controlled trial; RR: Relative risk; RTI: Respiratory tract infection}

\section{Acknowledgements}

Not applicable.

Funding

This study was undertaken with no funding.

Availability of data and materials

All data generated or analysed during this study are included in this published article.

Transparency declarations

None to declare.

\section{Authors' contributions}

AK contributed to the conception and design of the systematic review, the design and conduct of the search strategy, the quality assessment of the included trials, the trial selection and the acquisition, analysis, and interpretation of data and drafted the manuscript. FB contributed to the quality assessment of the included trials. SR contributed to the quality assessment of the included trials, the trial selection and to the conception of 
the search strategy. AH contributed to the conception and design of the systematic review. CL contributed to the design and conduct of the search strategy, the conception and design of the review as well as the interpretation of the data. AA contributed to the conception and design of the systematic review as well as the interpretation of the data. JFC contributed to the conception and design of the systematic review, the trial selection, the quality assessment of the included trials and the interpretation of the data. All authors read and approved the final manuscript.

\section{Ethics approval and consent to participate}

Not applicable.

\section{Consent for publication}

Not applicable.

\section{Competing interests}

The authors declare that they have no competing interests.

\section{Publisher's Note}

Springer Nature remains neutral with regard to jurisdictional claims in published maps and institutional affiliations.

\section{Author details}

'Clinic for Psychosomatic Medicine and Psychotherapy, University Medical Center, Rostock, Germany. ${ }^{2}$ Institute of General Practice, University Medical Center, Rostock, Germany. ${ }^{3}$ Department of Pediatric Pneumology, Immunology \& Intensive Care Medicine Charité, University Medical Center Berlin, Berlin, Germany. ${ }^{4}$ Institute for Biostatistics and Informatics in Medicine and Ageing Research, University Medical Center Rostock, Rostock, Germany. ${ }^{5}$ Institute for Community Medicine-Department of Family Medicine, University Greifswald, Greifswald, Germany. ${ }^{6}$ Institute of General Practice, University Medical Center, Rostock, Germany.

\section{Received: 4 April 2017 Accepted: 27 February 2018}

\section{Published online: 20 March 2018}

\section{References}

1. Holmes WF, Macfarlane JT, Macfarlane RM, Hubbard R. Symptoms, signs, and prescribing for acute lower respiratory tract illness. Br J Gen Pract. 2001;51:177-81.

2. Spinks A, Glasziou PP, Del Mar CB. Antibiotics for sore throat. Cochrane Database Syst Rev. 2013;11:CD000023.

3. Roca I, Akova M, Baquero F, Carlet J, Cavaleri M, Coenen S, et al. The global threat of antimicrobial resistance: science for intervention. New Microbes New Infect. 2015;6:22-9.

4. Sabuncu E, David J, Bernède-Bauduin C, Pépin S, Leroy M, Boëlle P-Y, et al. Significant reduction of antibiotic use in the community after a nationwide campaign in France, 2002-2007. PLoS Med. 2009;6:e1000084.

5. Rautakorpi U-M, Huikko S, Honkanen P, wKlaukka T, Mäkelä M, Palva E, et al. The Antimicrobial Treatment Strategies (MIKSTRA) Program: a 5year follow-up of infection-specific antibiotic use in primary health care and the effect of implementation of treatment guidelines. Clin Infect Dis 2006; 42:1221-1230.

6. Welschen I, Kuyvenhoven MM, Hoes AW, Verheij TJM. Effectiveness of a multiple intervention to reduce antibiotic prescribing for respiratory tract symptoms in primary care: randomised controlled trial. BMJ. 2004;329:431.

7. Coxeter P, Del Mar CB, McGregor L, Beller EM, Hoffmann TC Interventions to facilitate shared decision making to address antibiotic use for acute respiratory infections in primary care. Cochrane Database Syst Rev. 2015;11:CD010907.

8. Drekonja DM, Filice GA, Greer N, Olson A, MacDonald R, Rutks I, et al. Antimicrobial stewardship in outpatient settings: a systematic review. Infect Control Hosp Epidemiol. 2015;36:142-52.

9. World Health Organization. Global action plan on antimicrobial resistance. 2015 [cited 2017 Mar 13]; Available from: http://apps.who.int/iris/handle/ $10665 / 193736$

10. Arnold SR, Straus SE. Interventions to improve antibiotic prescribing practices in ambulatory care. Cochrane Database Syst Rev. 2005;4:CD003539.

11. Moher D, Liberati A, Tetzlaff J, Altman DG. Preferred Reporting Items for Systematic Reviews and Meta-Analyses: the PRISMA Statement. J Clin Epidemiol. 2009;62:1006-12.
12. Tates K, Meeuwesen L. Doctor-parent-child communication. A (re)view of the literature. Soc Sci Med. 2001:52:839-51.

13. Bjerrum L, Cots JM, Llor C, Molist N, Munck A. Effect of intervention promoting a reduction in antibiotic prescribing by improvement of diagnostic procedures: a prospective, before and after study in general practice. Eur J Clin Pharmacol. 2006;62:913-8.

14. Gjelstad S, Høye S, Straand J, Brekke M, Dalen I, Lindbæk M. Improving antibiotic prescribing in acute respiratory tract infections: cluster randomised trial from Norwegian general practice (prescription peer academic detailing (Rx-PAD) study). BMJ. 2013;347:f4403.

15. McGinn TG, McCullagh L, Kannry J, Knaus M, Sofianou A, Wisnivesky JP, et al. Efficacy of an evidence-based clinical decision support in primary care practices: a randomized clinical trial. JAMA Intern Med. 2013;173:1584-91.

16. Higgins JPT, Altman DG, Gøtzsche PC, Jüni P, Moher D, Oxman AD, et al. The Cochrane Collaboration's tool for assessing risk of bias in randomised trials. BMJ. 2011;d5928:343

17. Cohen J. Statistical power analysis for the behavioral sciences, vol. 2 Rev ed Hillsdale: Taylor \& Francis Inc; 1988.

18. Manager HCTPCT. Antibiotic use in outpatient settings [Internet]. [cited 2017 Nov 1]. Available from: http://pew.org/1N89p4C.

19. de With K, Schröder H, Meyer E. Antibiotikaanwendung in Deutschland im europäischen Vergleich. Dtsch Med Wochenschr. 2004;129:1987-92.

20. Altiner A, Brockmann S, Sielk M, Wilm S, Wegscheider K, Abholz H-H. Reducing antibiotic prescriptions for acute cough by motivating GPs to change their attitudes to communication and empowering patients: a cluster-randomized intervention study. J Antimicrob Chemother. 2007; 60:638-44.

21. Briel M, Langewitz W, Tschudi P, Young J, Hugenschmidt C, Bucher HC. Communication training and antibiotic use in acute respiratory tract infections. A cluster randomised controlled trial in general practice. Swiss Med Wkly. 2006;136:241-7.

22. Cals JWL, Butler CC, Hopstaken RM, Hood K, Dinant G-J. Effect of point of care testing for $C$ reactive protein and training in communication skills on antibiotic use in lower respiratory tract infections: cluster randomised trial. BMJ. 2009;338:b1374.

23. Gonzales R, Anderer T, McCulloch CE, Maselli JH, Bloom FJ, Graf TR, et al. A cluster-randomized trial of decision support strategies for reducing antibiotic use for acute bronchitis. JAMA Intern Med. 2013;173:267-73.

24. Linder JA, Schnipper JL, Tsurikova R, Yu T, Volk LA, Melnikas AJ, et al. Electronic health record feedback to improve antibiotic prescribing for acute respiratory infections. Am J Manag Care [Internet]. 2010;16. [cited 2017 Mar 14]. Available from: http://www.ajmc.com/journals/supplement/ 2010/ajmc_10dec_hit/ajmc_10hitdeclinder_exclu_e311

25. Linder JA, Schnipper JL, Tsurikova R, Yu T, Volk LA, Melnikas AJ, et al. Documentation-based clinical decision support to improve antibiotic prescribing for acute respiratory infections in primary care: a cluster randomised controlled trial. Inform Prim Care. 2009;17:231-40.

26. Little P, Stuart B, Francis N, Douglas E, Tonkin-Crine S, Anthierens S, et al. Effects of internet-based training on antibiotic prescribing rates for acute respiratory-tract infections: a multinational, cluster, randomised, factorial, controlled trial. Lancet Lond Engl. 2013;382:1175-82.

27. Andreeva $\mathrm{E}$, Melbye $\mathrm{H}$. Usefulness of $\mathrm{C}$-reactive protein testing in acute cough/respiratory tract infection: an open cluster-randomized clinical trial with C-reactive protein testing in the intervention group. BMC Fam Pract. 2014;15:80.

28. Gulliford MC, van Staa T, Dregan A, McDermott L, McCann G, Ashworth M, et al. Electronic health records for intervention research: a cluster randomized trial to reduce antibiotic prescribing in primary care (eCRT study). Ann Fam Med. 2014;12:344-51.

29. Meeker D, Linder JA, Fox CR, Friedberg MW, Persell SD, Goldstein NJ, et al. Effect of behavioral interventions on inappropriate antibiotic prescribing among primary care practices: a randomized clinical trial. JAMA. 2016;315:562-70

30. Cals JWL, Schot MJC, de Jong SAM, Dinant G-J, Hopstaken RM. Point-of-care C-reactive protein testing and antibiotic prescribing for respiratory tract infections: a randomized controlled trial. Ann Fam Med. 2010;8:124-33.

31. Llor C, Madurell J, Balagué M, Gómez M, Cots JM. Impact of rapid antigen detection testing on antibiotic prescription in acute pharyngitis in adults. A multicentric randomized controlled trial. Br J Gen Pract. 2011;61:e244-51.

32. Worrall G, Kettle A, Graham W, Hutchinson J. Postdated versus usual delayed antibiotic prescriptions in primary care: reduction in antibiotic use 
for acute respiratory infections? Can Fam Physician Med Fam Can. 2010;56: $1032-6$.

33. Hui Min Lee M, Shaw Teng Pan D, Huixin Huang J, I-Cheng Chen M, Hui Goh E, Jiang L. Efficacy of a patient-based health education intervention in reducing antibiotic use for acute upper respiratory tract infections in the private sector primary care setting in Singapore. Antimicrob Agents Chemother. 2017;61(5).

34. Cals JWL, de Bock L, Beckers P-JHW, Francis NA, Hopstaken RM, Hood K, et al. Enhanced communication skills and C-reactive protein point-of-care testing for respiratory tract infection: 3.5-year follow-up of a cluster randomized trial. Ann Fam Med. 2013;11:157-64.

35. Chalmers JD, Singanayagam A, Hill AT. C-reactive protein is an independent predictor of severity in community-acquired pneumonia. Am J Med. 2008; 121:219-25.

36. Hopstaken RM, Muris JW, Knottnerus JA, Kester AD, Rinkens PE, Dinant GJ. Contributions of symptoms, signs, erythrocyte sedimentation rate, and Creactive protein to a diagnosis of pneumonia in acute lower respiratory tract infection. Br J Gen Pract. 2003;53:358-64.

37. Tanz RR, Gerber MA, Kabat W, Rippe J, Seshadri R, Shulman ST. Performance of a rapid antigen-detection test and throat culture in community pediatric offices: implications for management of pharyngitis. Pediatrics. 2009;123: 437-44.

38. Spurling GKP, Del Mar CB, Dooley L, Foxlee R, Farley R. Delayed antibiotics for respiratory infections. Cochrane Database Syst Rev. 2013;4:CD004417.

39. Rubin MA, Bateman K, Alder S, Donnelly S, Stoddard GJ, Samore MH. A multifaceted intervention to improve antimicrobial prescribing for upper respiratory tract infections in a small rural community. Clin Infect Dis. 2005; 40:546-53.

40. Mölstad S, Erntell M, Hanberger H, Melander E, Norman C, Skoog G, et al. Sustained reduction of antibiotic use and low bacterial resistance: 10-year follow-up of the Swedish Strama programme. Lancet Infect Dis. 2008;8:125-32.

\section{Submit your next manuscript to BioMed Central and we will help you at every step:}

- We accept pre-submission inquiries

- Our selector tool helps you to find the most relevant journal

- We provide round the clock customer support

- Convenient online submission

- Thorough peer review

- Inclusion in PubMed and all major indexing services

- Maximum visibility for your research

Submit your manuscript at www.biomedcentral.com/submit 